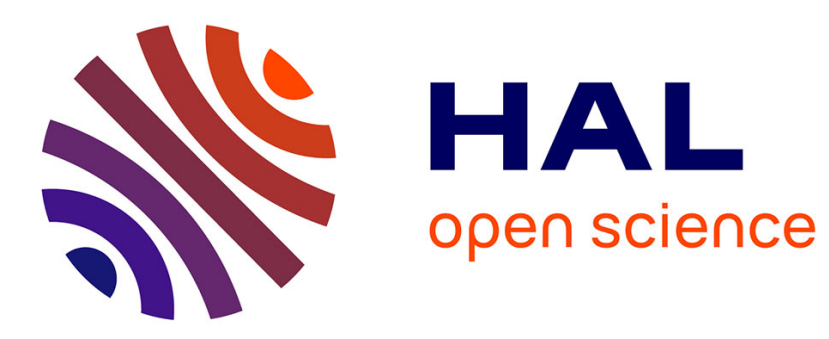

\title{
3D global optimal forcing and response of the supersonic boundary layer
}

Benjamin Bugeat, Jean-Camille Chassaing, Jean-Christophe Robinet, Pierre Sagaut

\section{- To cite this version:}

Benjamin Bugeat, Jean-Camille Chassaing, Jean-Christophe Robinet, Pierre Sagaut. 3D global optimal forcing and response of the supersonic boundary layer. Journal of Computational Physics, 2019, 398, pp.108888. 10.1016/j.jcp.2019.108888 . hal-02462825

\section{HAL Id: hal-02462825 \\ https://hal.science/hal-02462825}

Submitted on 31 Jan 2020

HAL is a multi-disciplinary open access archive for the deposit and dissemination of scientific research documents, whether they are published or not. The documents may come from teaching and research institutions in France or abroad, or from public or private research centers.
L'archive ouverte pluridisciplinaire HAL, est destinée au dépôt et à la diffusion de documents scientifiques de niveau recherche, publiés ou non, émanant des établissements d'enseignement et de recherche français ou étrangers, des laboratoires publics ou privés. 


\title{
Journal of Computational Physics
}

\section{D global optimal forcing and response of the supersonic boundary layer}

\author{
B. Bugeat ${ }^{\mathrm{a}, *}$, J.-C. Chassaing ${ }^{\mathrm{a}}$, J.-C. Robinet ${ }^{\mathrm{b}}$, P. Sagaut ${ }^{\mathrm{c}}$ \\ a Sorbonne Université, CNRS, Institut Jean Le Rond d'Alembert, F-75005 Paris, France \\ ${ }^{\mathrm{b}}$ DynFluid Laboratory - Arts et Métiers - 151, Bd. de l'Hôpital, 75013, Paris, France \\ c Aix Marseille Univ., CNRS, Centrale Marseille, M2P2 UMR 7340, 13451, Marseille, France
}

Keywords:

Optimal forcing

Global resolvent

Convective instability

Non-modal instability

Compressible boundary layer

\begin{abstract}
A B S T R A C T
3D optimal forcing and response of a 2D supersonic boundary layer are obtained by computing the largest singular value and the associated singular vectors of the global resolvent matrix. This approach allows to take into account both convective-type and component-type non-normalities responsible for the non-modal growth of perturbations in noise selective amplifier flows. It is moreover a fully non-parallel approach that does not require any particular assumptions on the baseflow. The numerical method is based on the explicit calculation of the Jacobian matrix proposed by Mettot et al. [1] for 2D perturbations. This strategy uses the numerical residual of the compressible Navier-Stokes equations imported from a finite-volume solver that is then linearised employing a finite difference method. Extension to 3D perturbations, which are expanded into modes of wave number, is here proposed by decomposing the Jacobian matrix according to the direction of the derivatives contained in its coefficients. Validation is performed on a Blasius boundary layer and a supersonic boundary layer, in comparison respectively to global and local results. Application of the method to a boundary layer at $M=4.5$ recovers three regions of receptivity in the frequency-transverse wave number space. Finally, the energy growth of each optimal response is studied and discussed.
\end{abstract}

\section{Introduction}

Depending on their dynamics, open flows can be divided into oscillator and noise selective amplifiers [2]. Whereas the first ones have an intrinsic dynamics related to the physical parameters of the baseflow, the second ones only amplify perturbations in specific ranges of frequencies, which grow in space and advected downstream. In terms of stability analysis, these considerations lead to distinguish absolute from convective instabilities. Local stability analysis [3] have extensively been employed to study the dynamics of various open flows (boundary layer [4], wakes [5], jet flows [6], etc.). This approach allows, in particular, to discriminate absolute and convective instabilities by computing the growth rate of zero group velocity waves [7]. The assumption of a (weakly) parallel baseflow is however required in order to expand perturbations into Fourier-Laplace modes along the streamwise direction.

\footnotetext{
* Corresponding author.

E-mail addresses: benjamin.bugeat@dalembert.upmc.fr (B. Bugeat), jean-camille.chassaing@sorbonne-universite.fr (J.-C. Chassaing), jean-christophe.robinet@ensam.eu (J.-C. Robinet), pierre.sagaut@univ-amu.fr (P. Sagaut).
} 


\begin{tabular}{|c|c|c|c|}
\hline$M$ & Mach number & $\infty$ & Far-field quantities \\
\hline $\operatorname{Pr}$ & Prandtl number & $\alpha_{r}$ & Streamwise wavenumber \\
\hline $\operatorname{Re}$ & Reynolds number & $\beta$ & Transverse wavenumber \\
\hline$x$ & Streamwise direction & $\omega$ & Angular frequency \\
\hline$y$ & Normal to the wall direction & $\Psi$ & Angle between the wave vector of the pertur- \\
\hline$z$ & $\begin{array}{l}\text { Transverse direction, supposed as homoge- } \\
\text { neous }\end{array}$ & $c_{\varphi}$ & $\begin{array}{l}\text { bation and the baseflow direction } \\
\text { Phase velocity }\end{array}$ \\
\hline $\mathcal{R}$ & Residual of Navier-Stokes equations & $\mu$ & Optimal gain \\
\hline $\mathbf{F}$ & $\begin{array}{l}\text { Flux of Navier-Stokes equations along } \\
x \text {-direction }\end{array}$ & $\widetilde{\mathbf{f}}$ & $\begin{array}{l}\text { Forcing vector (in particular, optimal forcing } \\
\text { vector) }\end{array}$ \\
\hline G & $\begin{array}{l}\text { Flux of Navier-Stokes equations along } \\
y \text {-direction }\end{array}$ & $\tilde{\mathbf{q}}$ & Perturbations vector (in particular, optimal re- \\
\hline $\mathbf{H}$ & $\begin{array}{l}\text { Flux of Navier-Stokes equations along } \\
z \text {-direction }\end{array}$ & $f_{X}$ & $\begin{array}{l}\text { sponse vector) } \\
\text { Streamwise forcing }\end{array}$ \\
\hline $\mathbf{q}$ & Vector of conservative variables $(\rho, \rho u, \rho v, \rho E)^{T}$ & $f_{y}$ & Normal forcing \\
\hline$u$ & Streamwise velocity & $f_{z}$ & Transverse forcing \\
\hline$v$ & Normal velocity & $\mathscr{J}$ & Jacobian matrix \\
\hline$w$ & Transverse velocity & $\mathscr{R}$ & Resolvent matrix \\
\hline$\rho$ & $\begin{array}{l}\text { Density } \\
\text { Speed of sound }\end{array}$ & $\mathbf{Q}_{E}$ & $\begin{array}{l}\text { Norm matrix associated with the energy of the } \\
\text { perturbations }\end{array}$ \\
\hline E & Total energy & $\mathbf{Q}_{F}$ & Norm matrix associated with the forcing field \\
\hline e & Internal energy & $\mathrm{d}_{\text {Chu }}$ & Chu's energy density profile \\
\hline$p$ & Pressure & $\mathrm{d}_{\mathrm{F}}$ & Forcing density profile \\
\hline$T$ & Temperature & $y_{\mathrm{m}}^{\mathrm{Chu}}$ & Ordinate where Chu's energy is maximum \\
\hline$\widehat{M}$ & Relative Mach number & $y_{\mathrm{m}}^{\mathrm{K}}$ & Ordinate where the kinetic energy is maxi- \\
\hline$\eta$ & Dynamic viscosity & & mum \\
\hline$\gamma$ & Heat capacity ratio & $\delta^{*}$ & Boundary layer compressible displacement \\
\hline$\kappa$ & Thermal conductivity & & thickness \\
\hline$c_{p}$ & Heat capacity & $\ell$ & Blasius length $\sqrt{\eta_{\infty} x / \rho_{\infty} u_{\infty}}$ \\
\hline
\end{tabular}

Focusing on the convectively unstable compressible boundary layer, first stability computations were based on a local approach [8-10]. Along with theoretical developments [11], these seminal studies established the main features of compressible instabilities, especially noting their inviscid nature caused by the existence of a generalised inflection point and the prevailing growth of 3D perturbations (Squire theorem [12] does not hold for compressible flows [9]). Later, local stability analysis allowed to suggest the existence of an additional unstable mode [13] (generally referred to as second mode, or Mack mode) in the case of sufficiently high supersonic Mach numbers $\left(M_{\infty} \geq 3.8\right)$, soon confirmed by experimental work $[14,15]$. Afterwards, more sophisticated local stability analysis taking into account the weak non-parallel effects produced more accurate results [16,17]. Following the work of Farrell [18] for incompressible flows, several local analysis then focused on computing non-modal growth for compressible boundary layer. Optimal growth in a temporal formulation was first proposed by Hanifi et al. [19] who were able to observe the non-modal growth of compressible streaks. A spatial version of this analysis was suggested by Tumin and Reshotko [20], afterwards improved by considering non-parallel effects [21,22] and 3D baseflows [23]. These approaches were coupled with a PSE method [24], resulting in a more general framework to study non-modal growth in weakly non-parallel flows $[25,26]$. However, these approaches can not be considered as universal as it does not allow to study fully non-parallel flows.

With the increase of computational resources, global stability analysis (in the sense of Theofilis [27]) became affordable. In this framework, the streamwise direction is solved as an eigen-direction which authorise to consider fully non-parallel baseflows. It offers a relevant tool to study globally unstable flows such as the bifurcations occurring in cavity flows [28] and shock wave/boundary layer interactions [29] or the onset of the transonic buffet on an airfoil [30]). Global stability analysis is however not suited to describe the dynamics of convectively unstable flows, which are globally stable. Instead, characterising the response of these flows subject to an external forcing constitutes a more relevant analysis as it is directly related to their noise amplifier nature [31]. In practice, this approach is related to the resolvent operator and an optimisation framework is employed to compute the optimal forcing and response for different frequencies. Such an analysis was first implemented for an incompressible boundary layer by performing a projection of the response onto a restricted number of global modes [32,33]. Another strategy was afterwards developed by Monokrousos et al. [34] using a time-stepping technique associated with an adjoint-based optimisation method. More recently, Sipp and Marquet [35] suggested to solve a singular value problem associated with the global resolvent operator and showed that, additionally, the left and right singular vectors constituted an orthonormal basis onto which the forcing and response fields could be expanded. Besides, 
it should be pointed out that these optimal response and forcing approaches are non-modal in nature. Indeed, the optimal response resulting from an optimisation problem can be seen as a superposition of global modes: both modal resonance and non-modal pseudo-resonance are thus taken into account [36]. These non-modal effects are a consequence of two types of non-normalities, associated with the non-normal nature of the linearised Navier-Stokes equations [37,38]. On the one hand, the convective-type non-normality (the term $(\overline{\rho \mathbf{U}} \cdot \nabla) \mathbf{u}^{\prime}$ in the linearised momentum equation), ubiquitous in convectively unstable flows, stems from the advection of perturbations by the baseflow. It was furthermore observed to cause a spatial separation of the forcing and response fields, respectively upstream and downstream [35]. On the other hand, the component-type or lift-up non-normality (the term $\left(\bar{\rho} \mathbf{u}^{\prime} \cdot \nabla\right) \overline{\mathbf{U}}$ in the linearised momentum equation) is caused by the transport of baseflow momentum by the perturbations. It was shown to produce component-wise transfer of energy between the forcing and response fields as in the case of the lift-up mechanism [39] or the Orr mechanism [40].

In compressible flows, a global approach taking into account non-modal effects was first implemented for jet flows as an optimal growth problem where an optimal initial condition was looked for [41,42]. Global optimal forcing based on resolvent computation was then developed and applied to the receptivity of a turbulent shock wave/boundary layer interaction [43]. However, to our knowledge, no work dealing with non-modal growth of 3D global perturbations in compressible flows has been published to date. Given that 3D convective instabilities are especially prevailing in this regime, an efficient numerical framework appears to be missing to tackle this problem.

In this paper, we propose a numerical method to study 3D global linear perturbations developing in convectively unstable, fully non-parallel, compressible 2D baseflows. This approach is based on the computation of the optimal gain and the associated optimal forcing and response, which is achieved by solving a singular value problem associated with the global resolvent operator [35]. The explicit numerical computation of the Jacobian matrix - the first step of the numerical method uses the discrete framework presented by Mettot et al. [1] which is here extended to 3D perturbation. This point constitutes the main original point of the present work and the mathematical derivation will be fully detailed. An application to the 3D receptivity of the supersonic boundary layer at $M=4.5$ is presented in order to demonstrate the potential of the method.

The paper is organised as follows. Governing equations and the theoretical approach involved in optimal gain computations are introduced in section 2. The numerical framework is developed in section 3, especially emphasising the computation of the 3D Jacobian matrix (section 3.3). Validation of the numerical framework is given in section 4 . Finally, a detailed study of the 3D receptivity of the supersonic boundary layer is presented in section 5 .

\section{Theoretical approach}

\subsection{Governing equations}

The flow is governed by the compressible Navier-Stokes equations. Variables are made non-dimensional according to

$$
\begin{gathered}
\widetilde{\mathbf{x}}=\frac{\mathbf{x}}{L}, \widetilde{t}=\frac{t}{L / u_{\infty}}, \widetilde{\rho}=\frac{\rho}{\rho_{\infty}}, \widetilde{\mathbf{u}}=\frac{\mathbf{u}}{u_{\infty}} \\
\widetilde{p}=\frac{p}{\rho_{\infty} u_{\infty}^{2}}, \widetilde{T}=\frac{T}{T_{\infty}}, \widetilde{E}=\frac{E}{u_{\infty}^{2}}, \widetilde{\eta}=\frac{\eta}{\eta_{\infty}}, \widetilde{\lambda}=\frac{\lambda}{\lambda_{\infty}}
\end{gathered}
$$

In the following, the $\sim$ symbol will be dropped in order to lighten notations. The $\infty$ symbol refers to far-field quantities. Conservative variables $\mathbf{q}=[\rho, \rho \mathbf{u}, \rho E]^{T}$ are used, where $\rho, \mathbf{u}=(u, v, w)^{T}$ and $E$ respectively are the fluid density, the velocity vector and the total energy. $T, p, \eta$ and $\lambda$ respectively stand for temperature, pressure, dynamic viscosity and thermal conductivity. The reference length $L$ may refer to the compressible boundary layer thickness $\delta^{*}$ or to the Blasius length $\ell=\sqrt{\eta_{\infty} X / \rho_{\infty} u_{\infty}}$. Non-dimensional Reynolds $R e$, Mach $M$ and Prandtl $\operatorname{Pr}$ numbers are introduced as

$$
R e=\frac{\rho_{\infty} u_{\infty} L}{\eta_{\infty}}, M=\frac{u_{\infty}}{c_{\infty}}, \operatorname{Pr}=\frac{\eta_{\infty} c_{p}}{\lambda_{\infty}}
$$

where $c$ is the speed of sound and $c_{p}$ is the heat capacity of the flow. The compressible Navier-Stokes equations can then be written as

$$
\begin{aligned}
& \frac{\partial \rho}{\partial t}+\nabla \cdot(\rho \mathbf{u})=0 \\
& \frac{\partial}{\partial t}(\rho \mathbf{u})+\nabla \cdot\left[\rho \mathbf{u} \otimes \mathbf{u}+p \mathbf{I}-\frac{1}{R e} \boldsymbol{\tau}\right]=0 \\
& \frac{\partial}{\partial t}(\rho E)+\nabla \cdot\left[(\rho E+p) \mathbf{u}-\frac{1}{\operatorname{Re}} \boldsymbol{\tau} \odot \mathbf{u}-\frac{\lambda}{\operatorname{PrRe}(\gamma-1) M^{2}} \nabla T\right]=0
\end{aligned}
$$

For a thermally and calorically perfect gas, the non-dimensional pressure $p$ and total energy $E$ can moreover be expressed according to

$$
p=\frac{1}{\gamma M_{\infty}^{2}} \rho T, E=\frac{p}{\rho(\gamma-1)}+\frac{1}{2} \mathbf{u} \cdot \mathbf{u}
$$


The viscous stress tensor of a Newtonian fluid is given by

$$
\boldsymbol{\tau}=\eta\left[\nabla \otimes \mathbf{u}+(\nabla \otimes \mathbf{u})^{T}-\frac{2}{3}(\nabla \cdot \mathbf{u}) \mathbf{I}\right]
$$

The dynamic viscosity is a function of temperature and is described by Sutherland's law [44]

$$
\eta(T)=T^{3 / 2} \frac{1+T_{s} / T_{\infty}}{T+T_{s} / T_{\infty}},
$$

where $T_{s}=110.4 \mathrm{~K}$ and $T_{\infty}=288 \mathrm{~K}$. The thermal conductivity coefficient also depends on the temperature in the same way as dynamic viscosity does $(\lambda(T) \sim \eta(T)$ [45]). Hence it leads to $\lambda=\eta$ as non-dimensional variables are used.

In the following, equations (3) can be recast in the dynamical system form

$$
\frac{\partial \mathbf{q}}{\partial t}=\mathcal{R}(\mathbf{q})
$$

where $\mathcal{R}$ is the differential nonlinear operator of Navier-Stokes equations.

\subsection{Linear dynamics}

This study aims to study the forced dynamics of 3D perturbations $\mathbf{q}^{\prime}(x, y, z, t)$ added to a $2 \mathrm{D}$ baseflow $\overline{\mathbf{q}}(x, y)$. The latter is a solution of the steady nonlinear compressible Navier-Stokes equations (3). Considering small amplitude perturbations and introducing a forcing term $\mathbf{f}^{\prime}(x, y, z, t)$, the governing equation of the perturbations is linear and written as

$$
\frac{\partial \mathbf{q}^{\prime}}{\partial t}=\mathscr{J} \mathbf{q}^{\prime}+\mathbf{f}^{\prime}
$$

where $\mathscr{J}=\partial \mathcal{R} /\left.\partial \mathbf{q}\right|_{\overline{\mathbf{q}}}$ is the Jacobian operator. The numerical computation of the Jacobian matrix will be described in section 3.3. In equation (8), the perturbations $\mathbf{q}^{\prime}$ can now be seen as the response of the flow to the external forcing $\mathbf{f}^{\prime}$ As $z$-direction is supposed to be homogeneous, these fields are expanded into Fourier modes of wave number $\beta$. Moreover, considering a harmonic forcing at frequency $\omega$, these quantities are finally written as

$$
\begin{aligned}
\mathbf{q}^{\prime}(x, y, z, t) & =\widetilde{\mathbf{q}}(x, y) e^{i(\beta z+\omega t)}+c . c . \\
\mathbf{f}^{\prime}(x, y, z, t) & =\widetilde{\mathbf{f}}(x, y) e^{i(\beta z+\omega t)}+c . c .
\end{aligned}
$$

Equation (8) can then be recast as

$$
\widetilde{\mathbf{q}}=\tilde{R} \mathbf{f}
$$

where $\mathscr{R}=(i \omega \mathscr{I}-\mathscr{J})^{-1}$ is the global resolvent operator (with $\mathscr{I}$ the identity operator) which depends both on the forcing frequency $\omega$ and the transverse wave number $\beta$. For globally stable flows, all eigenvalues of the Jacobian operator have a strictly negative real part. Thus, the resolvent operator is well defined and allows to study the forced dynamics of the flow by providing a relation between response and forcing fields. Optimal gain is now introduced and defined as the maximum ratio between the energy of the perturbations and the forcing. Formally, its expression reads

$$
\mu^{2}=\sup _{\widetilde{\mathbf{f}}} \frac{\|\widetilde{\mathbf{q}}\|_{E}^{2}}{\|\widetilde{\mathbf{f}}\|_{F}^{2}}
$$

where the energy norms $\|.\|_{E}$ and $\|.\|_{F}$ and the numerical computation of equation (12) will be described in section 3.5 .

\section{Numerical strategy}

\subsection{Compressible Navier-Stokes solver}

The baseflow is computed by means of a finite volume CFD solver as a steady solution of the nonlinear equations (3). Spatial discretisation of convective fluxes is performed using AUSM+ scheme [46] associated with a fifth-order MUSCL extrapolation [47]. Viscous fluxes at cell interfaces are obtained by a second-order centered finite difference scheme. The unsteady equations are marched in time until a steady state is reached. An implicit dual time stepping method with local time step is used [48]. This solver showed successful results in shock wave/boundary layer interaction computations [47]. In the present work, boundary layer baseflows are computed in a rectangular numerical domain (Fig. 1). A cartesian mesh is set with a geometrical progression from the wall. Boundary conditions are gathered in Table 1. Dirichlet and Neumann conditions are employed as only stationary solutions are computed. Besides, note that an adiabatic flate plate is considered. The length $L_{x_{0}}$ upstream from the leading edge (see Fig. 1) is set to zero when supersonic flow are considered. In subsonic computations, this length is set so that results do not depend on its value. Independence from the height $L_{y}$ of the domain is also checked in every baseflow and optimal gain computations. Validation of this solver for the case of a supersonic boundary layer at $M=4$ is provided in section 4.1 . 


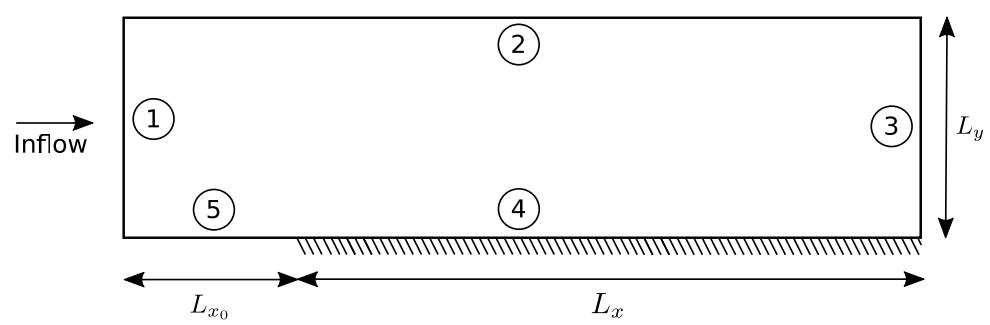

Fig. 1. Numerical domain.

Table 1

Boundary conditions used in baseflow computations.

\begin{tabular}{lll}
\hline Boundary & Supersonic conditions & Subsonic conditions \\
\hline 1 & $u=1, v=0, \rho=1, p=\frac{1}{\left(\gamma M^{2}\right)}$ & $u=1, v=0, \rho=1, \frac{\partial p}{\partial x}=0$ \\
2 & $\frac{\partial u}{\partial y}=0, \frac{\partial v}{\partial y}=0, \frac{\partial \rho}{\partial y}=0, p=\frac{1}{\left(\gamma M^{2}\right)}$ & $\frac{\partial u}{\partial y}=0, \frac{\partial v}{\partial y}=0, \frac{\partial \rho}{\partial y}=0, p=\frac{1}{\left(\gamma M^{2}\right)}$ \\
3 & $\frac{\partial u}{\partial x}=0, \frac{\partial v}{\partial x}=0, \frac{\partial \rho}{\partial x}=0, \frac{\partial p}{\partial x}=0$ & $\frac{\partial u}{\partial x}=0, \frac{\partial v}{\partial x}=0, \frac{\partial \rho}{\partial x}=0, p=\frac{1}{\left(\gamma M^{2}\right)}$ \\
4 & $u=0, v=0, \frac{\partial \rho}{\partial y}=0, \frac{\partial p}{\partial y}=0$ & $u=0, v=0, \frac{\partial \rho}{\partial y}=0, \frac{\partial p}{\partial y}=0$ \\
5 & $\frac{\partial u}{\partial y}=0, v=0, \frac{\partial \rho}{\partial y}=0, \frac{\partial p}{\partial y}=0$ & $\frac{\partial u}{\partial y}=0, v=0, \frac{\partial \rho}{\partial y}=0, \frac{\partial p}{\partial y}=0$
\end{tabular}

\subsection{Computation of the Jacobian matrix for $2 \mathrm{D}$ perturbations}

Before presenting the numerical strategy to compute the Jacobian matrix for 3D perturbations in section 3.3, the case of $2 \mathrm{D}$ perturbations $\mathbf{q}^{\prime}(x, y, z, t)=\widetilde{\mathbf{q}}(x, y) e^{i \omega t}+$ c.c. is first considered. Indeed, the former is actually an extension of the latter that was presented by Mettot [49]. The method is based on the linearisation of the 2D discretised equations. When dealing with compressible flows, provided that one owns a nonlinear CFD solver, this method allows to bypass the tedious linearisation and then discretisation of the compressible Navier-Stokes equations, thus reducing the risk of errors. Moreover, if one wants to compute adjoint quantities, working in a discretised framework may be more convenient [49].

From the discretisation of the system (7), whose dimension is $N \in \mathbb{N}$, the residual $\mathcal{R} \in \mathbb{R}^{N}$ of the $2 \mathrm{D}$ nonlinear NavierStokes equations is used to perform a first-order approximation of the Jacobian matrix as

$$
\mathscr{J} \mathbf{v}=\frac{\mathcal{R}(\overline{\mathbf{q}}+\varepsilon \mathbf{v})-\mathcal{R}(\overline{\mathbf{q}})}{\varepsilon}+O(\varepsilon)
$$

Here, $\overline{\mathbf{q}} \in \mathbb{R}^{N}$ is the vector of conservative variables associated with the baseflow, $\mathbf{v} \in \mathbb{R}^{N}$ is an arbitrary vector and $\varepsilon \in \mathbb{R}$ is a numerical parameter. The vector $\mathbf{v}$ is carefully chosen so that the coefficients of the matrix $\mathscr{J}$ can be conveniently recovered [49]. A simple approach consists in setting to zero every component of $\mathbf{v}$ except the $k$-component that is set to 1 (here, $k \in[1, N]$ ). Then, equation (13) allows to get every coefficient of the $k$-column of the matrix $\mathscr{J}$. Repeating this procedure $N$ times, the whole matrix is recovered. Moreover, it is possible to take advantage from the block diagonal structure of $\mathscr{J}$ in order to speed up the method. As described by Mettot [49], the vector $\mathbf{v}$ can be filled with more than one component equal to 1 . This leads to reduce the number of calls to equation (13) by approximatively 100 . This efficiency actually depends on the order of the numerical scheme and on the proportion of points in the normal and streamwise direction. As an example, computing the matrix $\mathscr{J}$ when $N=600000$ using a $1500 \times 100$ mesh and a third order accurate scheme takes approximatively 5 minutes (on CPU: Intel Xeon(R) CPU E5-2630v2@ 2.60GHz). As for the choice of $\varepsilon$ value, its order of magnitude has to be small enough so that second order terms can be neglected but need to be high enough to avoid numerical round-off errors. If the vector $\mathbf{v}$ contains only one non-zero element located at the $k$-component, Knoll and Keyes [50] suggest to chose $\varepsilon=b\left(1+\left|\bar{q}_{k}\right|\right)$, where $b$ is the square root of the machine precision. If $\mathbf{v}$ contains multiple non-zero components, then an average of this expression can be used. However, in practice, setting a fixed value of $\varepsilon$ between $10^{-6}$ and $10^{-8}$ was observed to be a robust choice, probably thanks to the use of nondimensional quantities [50].

\subsection{Extension to $3 D$ perturbations}

The method employed to compute a Jacobian matrix in a discretised-then-linearised framework presented in the previous section is now extended to 3D perturbations $\mathbf{q}^{\prime}(x, y, z, t)=\widetilde{\mathbf{q}}(x, y) e^{i(\beta z+\omega t)}$. In the present work, the baseflow is supposed to be homogeneous in the $z$-direction so that

$$
\forall(x, y) \in \mathscr{V}, \frac{\partial \overline{\mathbf{q}}}{\partial z}=0 \text { and } \bar{w}=0
$$


where $\mathscr{V}$ is the computational domain. The proposed approach lies in the use of 3D Navier-Stokes residual (provided by a finite-volume solver) to perform the first-order approximation in equation (13). However, the $z$-direction must now be treated as a Fourier direction, which forbids the direct use of this approximation because transverse derivatives $\partial / \partial z$ must be turned into $i \beta$ terms. In order to apply a special treatment in this direction, let us first write equation (3) with fluxes as

$$
\frac{\partial \mathbf{q}}{\partial t}=\mathcal{R}(\mathbf{q})=-\frac{\partial \mathbf{F}}{\partial x}-\frac{\partial \mathbf{G}}{\partial y}-\frac{\partial \mathbf{H}}{\partial z}
$$

where each flux term is now separated in two parts as follows

$$
\begin{aligned}
& \mathbf{F}=\mathbf{F}^{\prime}-\mathbf{F}_{v z} \\
& \mathbf{G}=\mathbf{G}^{\prime}-\mathbf{G}_{v z} \\
& \mathbf{H}=\mathbf{H}^{\prime}-\mathbf{H}_{v z}
\end{aligned}
$$

The part with subscript $v z$ includes every transverse derivatives $\partial / \partial z$, which are only of viscous nature. The other part (superscript ') contains the remaining terms. Explicit expressions of these fluxes are given in Appendix A. The Jacobian matrix is finally separated into three different matrices as

$$
\mathscr{J}=\mathscr{J}_{F, G}^{\prime}+\mathscr{J}_{H}^{\prime}+\mathscr{J}_{v z}
$$

where

$$
\begin{aligned}
\mathscr{J}_{F, G}^{\prime} & =\frac{\partial}{\partial \mathbf{q}}\left[-\left(\frac{\partial \mathbf{F}^{\prime}}{\partial x}+\frac{\partial \mathbf{G}^{\prime}}{\partial y}\right)\right]_{\overline{\mathbf{q}}} \\
\mathscr{J}_{H}^{\prime} & =\frac{\partial}{\partial \mathbf{q}}\left[-\frac{\partial \mathbf{H}^{\prime}}{\partial z}\right]_{\overline{\mathbf{q}}} \\
\mathscr{J}_{v z} & =\frac{\partial}{\partial \mathbf{q}}\left[\frac{\partial \mathbf{F}_{\nu z}}{\partial x}+\frac{\partial \mathbf{G}_{\nu z}}{\partial y}+\frac{\partial \mathbf{H}_{\nu z}}{\partial z}\right]_{\overline{\mathbf{q}}}
\end{aligned}
$$

Each matrix is then numerically computed by a specific method that is detailed in the following subsections.

3.3.1. Computation of $\mathscr{J}_{F, G}^{\prime}$

No transverse derivative is involved in the computation of $\mathscr{J}_{F, G}^{\prime}$ in equation (20). Therefore, equation (13) can be straightforwardly used by introducing $\mathcal{R}_{F, G}^{\prime}=-\partial \mathbf{F}^{\prime} / \partial x-\partial \mathbf{G}^{\prime} / \partial y$. The Jacobian matrix $\mathscr{J}_{F, G}^{\prime}$ is thus computed according to

$$
\mathscr{J}_{F, G}^{\prime} \mathbf{v}=\frac{\mathcal{R}_{F, G}^{\prime}(\overline{\mathbf{q}}+\varepsilon \mathbf{v})-\mathcal{R}_{F, G}^{\prime}(\overline{\mathbf{q}})}{\varepsilon}
$$

\subsubsection{Computation of $\mathscr{J}_{H}^{\prime}$}

The computation of $\mathscr{J}_{H}^{\prime}$ is achieved by firstly reconsidering the following linearisation

$$
\mathscr{J}_{H}^{\prime} \mathbf{q}^{\prime}=-\frac{\partial \mathbf{H}^{\prime}\left(\overline{\mathbf{q}}+\mathbf{q}^{\prime}\right)}{\partial z}+\frac{\partial \mathbf{H}^{\prime}(\overline{\mathbf{q}})}{\partial z}
$$

where a first order expansion of $\mathbf{H}^{\prime}\left(\overline{\mathbf{q}}+\mathbf{q}^{\prime}\right)$ in the first right hand term gives

$$
\mathscr{J}_{H}^{\prime} \mathbf{q}^{\prime}=-\frac{\partial}{\partial z}\left[\mathbf{H}^{\prime}(\overline{\mathbf{q}})+\left.\frac{\partial \mathbf{H}^{\prime}}{\partial \mathbf{q}}\right|_{\overline{\mathbf{q}}} \mathbf{q}^{\prime}\right]+\frac{\partial \mathbf{H}^{\prime}(\overline{\mathbf{q}})}{\partial z}
$$

Finally, the following expression remains

$$
\mathscr{J}_{H}^{\prime} \mathbf{q}^{\prime}=-\frac{\partial}{\partial z}\left[\left.\frac{\partial \mathbf{H}^{\prime}}{\partial \mathbf{q}}\right|_{\overline{\mathbf{q}}} \mathbf{q}^{\prime}\right]
$$

Here, $\partial \mathbf{H}^{\prime} /\left.\partial \mathbf{q}\right|_{\overline{\mathbf{q}}}$ does not depend on $z$ as assumed in equation (14). And since $\partial \mathbf{q}^{\prime} / \partial z=i \beta \mathbf{q}^{\prime}$, equation (26) now reads

$$
\mathscr{J}_{H}^{\prime} \mathbf{q}^{\prime}=-\left.i \beta \frac{\partial \mathbf{H}^{\prime}}{\partial \mathbf{q}}\right|_{\overline{\mathbf{q}}} \mathbf{q}^{\prime}
$$

The expression of $\mathscr{J}_{H}^{\prime}$ is finally identified as 


$$
\mathscr{J}_{H}^{\prime}=-\left.i \beta \frac{\partial \mathbf{H}^{\prime}}{\partial \mathbf{q}}\right|_{\overline{\mathbf{q}}}
$$

Again, the numerical computation of $\partial \mathbf{H}^{\prime} /\left.\partial \mathbf{q}\right|_{\overline{\mathbf{q}}}$ is based on a first-order finite difference approximation. However, note that the numerical flux $\mathbf{H}^{\prime}$ is here used instead of the flux divergence that was previously needed in equation (13) and (23). Hence, the matrix $\mathscr{J}_{H}^{\prime}$ is numerically built according to

$$
\mathscr{J}_{H}^{\prime} \mathbf{v}=-i \beta \frac{\mathbf{H}^{\prime}(\overline{\mathbf{q}}+\varepsilon \mathbf{v})-\mathbf{H}^{\prime}(\overline{\mathbf{q}})}{\varepsilon}
$$

\subsubsection{Computation of $\mathscr{J}_{v z}^{\prime}$}

Because every flux term now contains a transverse derivative, a particular care is required to compute the matrix $\mathscr{J}_{v z}^{\prime}$. It is not simply possible to replace each $\partial / \partial z$ into $i \beta$ within the fluxes $\mathbf{F}_{v z}, \mathbf{G}_{v z}$ and $\mathbf{H}_{v z}$. Indeed, spurious non-zero terms associated with the baseflow derivative along $z$ would appear, thus violating the assumptions made in equations (14). To get around this problem, it can be observed that because of equations (14), the perturbations $\widetilde{\mathbf{q}}$ only appears under $z$-derivatives in the final linearised equations. For example, introducing the linearisation operator $\mathscr{L}$, linearising the fourth component of the vector $\mathbf{F}_{v z}$ (see Appendix A) reads

$$
\mathscr{L}\left(\frac{\eta}{\operatorname{Re}} \frac{\partial u}{\partial z}\right)=\frac{\bar{\eta}}{\operatorname{Re}} \frac{\partial \widetilde{u}}{\partial z}=i \beta \frac{\bar{\eta}}{\operatorname{Re}} \widetilde{u}
$$

Anticipating the final result of linearisation, we here suggest to modify the fluxes $\mathbf{F}_{v z}, \mathbf{G}_{v z}$ and $\mathbf{H}_{v z}$ into $\widehat{\mathbf{F}}_{v z}, \widehat{\mathbf{G}}_{v z}$ and $\widehat{\mathbf{H}}_{v z}$ in which every factor in front of a $z$-derivative is set to the baseflow value and each $\partial / \partial z$ is turned into $i \beta$ (which will appear as a factor in the final expression of the Jacobian matrix). To illustrate this procedure, let us take the example of $\mathbf{F}_{\nu z}$ (see Appendix B for exhaustive expressions of the modified fluxes)

$$
\mathbf{F}_{v z}=\left(\begin{array}{c}
0 \\
-\frac{2}{3} \frac{\eta}{R e} \frac{\partial w}{\partial z} \\
0 \\
\frac{\eta}{R e} \frac{\partial u}{\partial z} \\
\frac{\eta}{R e}\left[-u \frac{2}{3} \frac{\partial w}{\partial z}+w \frac{\partial u}{\partial z}\right]
\end{array}\right) \rightarrow \widehat{\mathbf{F}}_{v z}=\left(\begin{array}{c}
0 \\
-\frac{2}{3} \frac{\bar{\eta}}{R e} w \\
0 \\
\frac{\bar{\eta}}{R e} u \\
\overline{\frac{\eta}{R e}}\left[-\bar{u} \frac{2}{3} w+\bar{w} u\right]
\end{array}\right)
$$

The matrix $\mathscr{J}_{v z}^{\prime}$ is finally computed using the approaches presented in section 3.3.1 for $\widehat{\mathbf{F}}_{v z}$ and $\widehat{\mathbf{G}}_{v z}$ and in section 3.3.2 for $\widehat{\mathbf{H}}_{v z}$. Introducing

$$
\widehat{\mathcal{R}}_{v z}=\frac{\partial \widehat{\mathbf{F}}_{v z}}{\partial x}+\frac{\partial \widehat{\mathbf{G}}_{v z}}{\partial y}
$$

the final practical expression to compute $\mathscr{J}_{v z}^{\prime}$ reads

$$
\mathscr{J}_{\nu z}^{\prime} \mathbf{v}=i \beta \frac{\widehat{\mathcal{R}}_{v z}(\overline{\mathbf{q}}+\varepsilon \mathbf{v})-\widehat{\mathcal{R}}_{\nu z}(\overline{\mathbf{q}})}{\varepsilon}-\beta^{2} \frac{\widehat{\mathbf{H}}_{v z}(\overline{\mathbf{q}}+\varepsilon \mathbf{v})-\widehat{\mathbf{H}}_{v z}(\overline{\mathbf{q}})}{\varepsilon}
$$

\subsection{Conclusion about 3D extension}

It should finally be noted that the numerical implementation of the 3D extension from the 2D method is straightforward, provided that 3D Navier-Stokes residual is available. Indeed, 3D extension needs minor modifications of the numerical fluxes but recovering the coefficients of the Jacobian matrix, which constitutes the main effort of numerical implementation (see section 3.2), is achieved by directly using the $2 \mathrm{D}$ computation routine. Here is a summary of the main steps to compute the 3D Jacobian matrix from an already implemented 2D method (aside from redefinitions of numerical arrays to take into account the additional transverse momentum component).

1. Import 3D Navier-Stokes residual routines.

2. Modify the fluxes within these routines according to Appendix B.

3. Using equations (23), (29) and (33), compute

$$
\mathscr{J} \mathbf{v}=\mathscr{J}_{F, G}^{\prime} \mathbf{v}+\mathscr{J}_{H}^{\prime} \mathbf{v}+\mathscr{J}_{v z}^{\prime} \mathbf{v}
$$

4. Recover the coefficients using the method presented in section 3.2.

It should be finally pointed out that additional computational costs, in terms of random-access memory, is kept affordable compare to the 2D method (in practice, a 50\% increase tends to be observed). Indeed, introducing a fifth component 
(transverse velocity) automatically increases the storage, but because a Fourier expansion is used in the transverse direction, no discretisation is performed in this direction. Conversely, implementing a fully 3D method (necessary for 3D baseflows) would dramatically increase computations costs. Indeed, the number of coefficients in the Jacobian matrix would increase linearly with the number of points $N_{z}$ in the transverse direction. Worst, the storage needed to solve the linear systems that will be introduced in section 3.5 would scale as $N_{z}^{2}$. If it seems today possible to achieve such computations using large clusters and limited numbers of points, the method presented in this paper offers a significantly more efficient way to compute 3D perturbations when considering 2D baseflows.

\subsection{Optimal forcing and response computation}

This section presents the numerical approach proposed by [35] to compute the optimal gain defined in equation (12) and the associated optimal forcing and response fields $\widetilde{\mathbf{f}}(x, y)$ and $\widetilde{\mathbf{q}}(x, y)$. The resolvent matrix $\mathscr{R}$ is involved in this problem and first requires the computation of the Jacobian matrix introduced in the previous section. Note that the explicit construction of $\mathscr{R}$ will not be required since linear systems involving $\mathscr{R}^{-1}$ will be solved.

Equation (12) is an optimisation problem on the function $\mu(\widetilde{\mathbf{f}})$ which depends on energy norms. These norms are associated with discrete scalar products that can be expressed by norm matrices as

$$
\begin{gathered}
\|\widetilde{\mathbf{q}}\|_{E}^{2}=<\widetilde{\mathbf{q}}, \widetilde{\mathbf{q}}>_{E}=\widetilde{\mathbf{q}}^{*} \mathbf{Q}_{E} \widetilde{\mathbf{q}} \\
\|\widetilde{\mathbf{f}}\|_{F}^{2}=<\widetilde{\mathbf{f}} \widetilde{\mathbf{f}}>_{F}=\widetilde{\mathbf{f}}^{*} \mathbf{Q}_{F} \widetilde{\mathbf{f}}
\end{gathered}
$$

where ${ }^{*}$ stands for the transconjugate operator. The choice of $\mathbf{Q}_{E}$ in equation (35) is related to the energy of the perturbations that one wants to optimise. For incompressible flows, considering the kinetic energy appears as a natural choice [18]. For compressible flows, Chu's energy norm [51] (also called Mack's norm) is widely used to study the non-modal behaviour of compressible flow dynamics [19,20,25]. It contains the kinetic energy of the perturbations and a strictly positive term relative to thermodynamical perturbations. As such, Chu's energy is necessarily greater than or equal to kinetic energy. Explicit expression of the norm matrix $\mathbf{Q}_{E}=\mathbf{Q}_{\mathrm{Chu}}$ associated with Chu's energy and written for conservative variables is derived hereafter, whereas $\mathbf{Q}_{E}=\mathbf{Q}_{\mathrm{EK}}$, associated with the kinetic energy, is detailed in Appendix C. Starting off from primitive variables, Chu's disturbances energy $E_{\text {Chu }}$ reads [51]

$$
E_{\mathrm{Chu}}=\frac{1}{2} \int_{\mathscr{V}}\left(\bar{\rho}\left|\mathbf{u}^{\prime}\right|^{2}+\frac{\bar{T}}{\bar{\rho} \gamma M^{2}}\left(\rho^{\prime}\right)^{2}+\frac{\bar{\rho}}{(\gamma-1) \gamma M^{2} \bar{T}}\left(T^{\prime}\right)^{2}\right) \mathrm{d} \Omega
$$

The norm matrix $\mathbf{Q}_{\text {chu }}$ is then defined such that

$$
\int_{\mathscr{V}} \mathbf{q}^{\prime *} \mathbf{Q}_{\mathrm{Chu}} \mathbf{q}^{\prime}=E_{\mathrm{Chu}}
$$

where $\mathbf{q}^{\prime}$ is the state vector of perturbations written as conservative variables. Let us recall that physical variables are here made dimensionless following equations (1). Then, primitive variables can be translated into conservative variables by the following relations

$$
\begin{aligned}
u_{i}^{\prime} & =\frac{1}{\bar{\rho}}\left(\left(\rho u_{i}\right)^{\prime}-\overline{u_{i}} \rho^{\prime}\right) \\
T^{\prime} & =\frac{(\gamma-1) \gamma M^{2}}{\bar{\rho}}\left(\left(\frac{1}{2}|\overline{\mathbf{u}}|^{2}-\bar{e}\right) \rho^{\prime}-\overline{u_{i}}\left(\rho u_{i}\right)^{\prime}+(\rho E)^{\prime}\right)
\end{aligned}
$$

where $e$ is the internal energy of the flow. Two parameters, associated with baseflow quantities, are now introduced as

$$
\begin{aligned}
& a_{1}=\frac{(\gamma-1) \gamma M^{2} \bar{\rho}}{\bar{T}} \\
& a_{2}=\frac{\left(\frac{1}{2}|\overline{\mathbf{u}}|^{2}-\bar{e}\right)}{\bar{\rho}}
\end{aligned}
$$

Equation (37) can now be recast with conservative variables. Identifying this equation with equation (38) and searching the matrix $Q_{\text {Chu }}$ as symmetrical, its coefficients can be identified as 


$$
\mathbf{Q}_{\text {chu }}=\frac{1}{2} \mathrm{~d} \Omega\left(\begin{array}{ccccc}
\frac{|\overline{\mathbf{u}}|^{2}}{\bar{\rho}}+\frac{\bar{T}}{\bar{\rho} \gamma M^{2}}+a_{1} a_{2}^{2} & -\frac{\bar{u}\left(1+a_{1} a_{2}\right)}{\bar{\rho}} & -\frac{\bar{v}\left(1+a_{1} a_{2}\right)}{\bar{\rho}} & 0 & \frac{a_{1} a_{2}}{\bar{\rho}} \\
-\frac{\bar{u}\left(1+a_{1} a_{2}\right)}{\bar{\rho}} & \frac{1}{\bar{\rho}}+\frac{\bar{u}^{2} a_{1}}{\overline{\rho^{2}}} & \frac{\overline{u v} a_{1}}{\bar{\rho}^{2}} & 0 & -\frac{\bar{u} a_{1}}{\bar{\rho}^{2}} \\
-\frac{\bar{v}\left(1+a_{1} a_{2}\right)}{\bar{\rho}} & \frac{\overline{u v} a_{1}}{\bar{\rho}^{2}} & \frac{1}{\bar{\rho}}+\frac{\bar{v}^{2} a_{1}}{\bar{\rho}^{2}} & 0 & -\frac{\bar{v} a_{1}}{\bar{\rho}^{2}} \\
0 & 0 & 0 & \frac{1}{\bar{\rho}} & 0 \\
\frac{a_{1} a_{2}}{\bar{\rho}} & -\frac{\bar{u} a_{1}}{\bar{\rho}^{2}} & -\frac{\bar{v} a_{1}}{\bar{\rho}^{2}} & 0 & \frac{a_{1}}{\bar{\rho}^{2}}
\end{array}\right)
$$

Numerically, a first-order integration over the numerical domain $\Omega$ is performed. To do so, a block diagonal matrix is built from the matrix in equation (43), taking care of setting $\mathrm{d} \Omega_{i, j}$ and baseflow values for each elementary volume.

The matrix $\mathbf{Q}_{F}$ in equation (36) is defined from the canonical scalar product $\|\widetilde{\mathbf{f}}\|_{F}^{2}=\int_{\Omega} \widetilde{\mathbf{f}} \widetilde{\mathbf{f}} \mathbf{d} \Omega$ (see Appendix $C$ for explicit expression). This matrix is positive definite. It can be noted that $\left.|| \widetilde{\mathbf{f}}\right|_{F} ^{2}$ is not homogeneous to an energy, but it is rather a mathematical norm which is chosen in order to reflect the energy input of the external forcing field $0 .{ }^{1}$ Hence the optimal gain is not strictly defined as a ratio of two energies, and its absolute value has no physical meaning. However, detecting maximum values of $\mu$ relative to different wave numbers and frequencies still allows to find out resonance and pseudo-resonance of the flow response to a harmonic forcing. As such, it remains a relevant tool to analyse the linear dynamics of a noise selective amplifier flow.

It is possible to constrain the forcing field both to a localised region of the flow and to specific components by introducing a matrix $\mathbf{P}$ such that $\widetilde{\mathbf{f}}=\mathbf{P} \widetilde{\mathbf{f}_{\mathbf{s}}}$. In this paper, we choose to only consider the momentum components of the forcing field in order to simplify the interpretation of the forcing norm. A similar choice has been made for example by Sartor et al. [43]. In this case the matrix $\mathbf{P}$ has, before discretisation, the following expression

$$
P=\left(\begin{array}{lll}
0 & 0 & 0 \\
1 & 0 & 0 \\
0 & 1 & 0 \\
0 & 0 & 1 \\
0 & 0 & 0
\end{array}\right)
$$

If $N$ is the size of the vector $\widetilde{\mathbf{f}}$, then $\widetilde{\mathbf{f}_{\mathbf{s}}}$ has a size $M$ with $M \leq N$ and the matrix $\mathbf{P}$ has a size $N \times M$. The relation between forcing and response fields then reads

$$
\tilde{\mathbf{q}}=\mathscr{R} \mathbf{P \mathbf { f } _ { S }}
$$

Introducing energy norm matrices, equation (12) can now be recast as

$$
\mu^{2}=\sup _{\widetilde{\mathbf{f}}_{s}} \frac{\left(\mathscr{R} \mathbf{P} \tilde{\mathbf{f}}_{s}\right)^{*} \mathbf{Q}_{\mathbf{E}}\left(\mathscr{R} \tilde{\mathbf{P f}}_{s}\right)}{\left(\widetilde{\mathbf{P f}}_{s}\right)^{*} \mathbf{Q}_{\mathbf{F}}\left(\widetilde{\mathbf{P f}}_{s}\right)}=\sup _{\widetilde{\mathbf{f}}_{s}} \frac{\left(\widetilde{\mathbf{P f}}_{s}\right)^{*} \mathscr{R} \mathbf{Q}_{\mathbf{E}} \mathscr{R}\left(\tilde{\mathbf{P f}}_{s}\right)}{\left(\widetilde{\mathbf{P f}}_{s}\right)^{*} \mathbf{Q}_{\mathbf{F}}\left(\widetilde{\mathbf{P f}}_{s}\right)}
$$

Equation (46) can be seen as a generalized Rayleigh quotient [35] where the optimal gain $\mu^{2}$ is then the largest eigenvalue and $\widetilde{\mathbf{f}_{\mathbf{s}}}$ the associated eigenvector of the Hermitian eigenvalue problem

$$
\left(\mathscr{R}^{*} \mathbf{Q}_{\mathbf{E}} \mathscr{R} \mathbf{P}\right) \tilde{\mathbf{f}}_{s}=\mu^{2}\left(\mathbf{Q}_{\mathbf{F}} \mathbf{P}\right) \widetilde{\mathbf{f}}_{s}
$$

Because $\mathbf{Q}_{\mathbf{F}}$ is invertible and $\mathbf{P}^{*} \mathbf{P}=\mathbf{I}$, equation (47) reduces to

$$
\underbrace{\left(\mathbf{P}^{*} \mathbf{Q}_{\mathbf{F}}^{-1} \mathscr{R}^{*} \mathbf{Q}_{\mathbf{E}} \mathscr{R} \mathbf{P}\right)}_{\mathbf{A}} \tilde{\mathbf{f}}_{s}=\mu^{2} \widetilde{\mathbf{f}}_{s}
$$

To solve equation (48), only the inverse of the resolvent matrix $\mathscr{R}$, which can conveniently be computed from the Jacobian matrix, is actually required. Indeed, the eigenvalue problem (48) can be solved by a matrix-free algorithm based on a Krylov method [52]. A set of vectors $\left(\mathbf{v}_{0}, \mathbf{A v}_{0}, \mathbf{A}^{2} \mathbf{v}_{0}, \ldots\right)$, which composes the Krylov subspace associated with the matrix $\mathbf{A}$, needs to be computed. Starting from an arbitrary vector $\mathbf{v}_{0}$, each Krylov vector $\mathbf{v}_{i}$ is computed from the previous one $\mathbf{v}_{i-1}$ by solving equation $\mathbf{v}_{i}=\mathbf{A} \mathbf{v}_{i-1}$. This computation requires to solve two linear systems involving $\mathscr{R}^{-1}$ and $\left(\mathscr{R}^{*}\right)^{-1}$. The detailed steps of this procedure are developed in Algorithm 1. Finally, the optimal response can be recovered by solving the linear system (45).

\footnotetext{
1 Note that defining an input mechanical work would be more rigorous, but, to our knowledge, this can only be achieved a posteriori of the optimal gain computation. This point has been thoroughly discussed by Sipp and Marquet [35].
} 


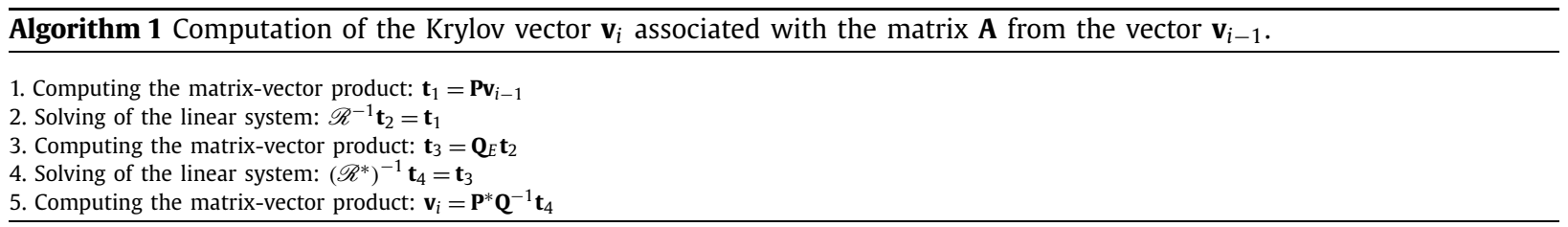

Open library PETSc [53] interfaced with MUMPS [54] is used to solve the linear systems by a direct sparse LU algorithm. The matrix-free eigenvalue problem is solved using SLEPc library [55] by a Krylov-Schur algorithm [56].

\subsection{Boundary conditions on perturbations}

Table 2 gathers the Dirichet-Neumann conditions that are applied on perturbations at each boundary. Numerically, a matrix $\mathscr{B}$ is introduced in the equations (8) such that

$$
\mathscr{B} \frac{\partial \mathbf{q}^{\prime}}{\partial t}=\mathscr{J} \mathbf{q}^{\prime}+\mathbf{f}^{\prime}
$$

The matrix $\mathscr{B}$ is defined as the identity matrix except that diagonal coefficients are set to zero at lines corresponding to boundary points. Note that, in practice, the global resolvent matrix is hence defined as $\mathscr{R}=(i \omega \mathscr{B}-\mathscr{J})^{-1}$. In order to finally implement the conditions given in Table 2, the coefficients of the Jacobian matrix are directly set without using the procedure given by equation (13).

\begin{tabular}{|c|c|}
\hline Boundary & Conditions \\
\hline 1 & $u^{\prime}=0, v^{\prime}=0, \rho^{\prime}=0, p^{\prime}=0$ \\
\hline 2 & $u^{\prime}=0, \frac{\partial v}{\partial y}=0, \rho^{\prime}=0, p^{\prime}=0$ \\
\hline 3 & $\frac{\partial u^{\prime}}{\partial x}=0, \frac{\partial v^{\prime}}{\partial x}=0, \frac{\partial \rho^{\prime}}{\partial x}=0, \frac{\partial p^{\prime}}{\partial x}=0$ \\
\hline 4 & $u^{\prime}=0, v^{\prime}=0, \frac{\partial \rho}{\partial y}=0, \frac{\partial p}{\partial y}=0$ \\
\hline
\end{tabular}

\section{Validation of the present method}

In this section, solvers presented in section 3 are validated against data from existing studies. Solutions from the CFD solver are compared to the self-similar solution of the compressible boundary layer. Optimal gain computations are first validated against 3D global results for a Blasius boundary layer. Afterwards, a validation against 3D non-global results for a supersonic boundary layer is performed since no results for 3D global optimal perturbations are known for compressible flows (as opposed to 3D global stability results for compressible flows, for example presented by Hildebrand et al. [57]). Note that because three different test cases have been considered, Mach numbers and Reynolds numbers vary from one case to another accordingly with the existing data found in the literature. These configurations are gathered in Table 3.

Table 3

Flows studied in section 4 to validate CFD and optimal gain solvers. Reynolds numbers are given as presented in the existing studies.

\begin{tabular}{lllll}
\hline$\S$ & Validation purpose & Reference & Mach & Reynolds at inflow/outflow \\
\hline 4.1 & Non-linear solution & {$[58]$} & $M=4$ & self similar solution \\
4.2 & 3D global perturbations & {$[34]$} & $M=0.3$ & $\operatorname{Re}_{\delta^{*}}=1000 / 1836$ \\
4.3 & 3D non-global perturbations & {$[21]$} & $M=3$ & $\operatorname{Re}_{\ell}=0 / 1000$ \\
\hline
\end{tabular}

\subsection{CFD solver validation}

A computation of a supersonic boundary layer flow is performed at $M=4$ in order to assess the baseflow solver presented in section 3.1. The numerical domain starts at the leading edge of the assumed adiabatic flat plate. The Reynolds number at outflow is set to $R e_{x}=2 \times 10^{6}$. The steady nonlinear Navier-Stokes equations (3) are solved on a mesh of $800 \times$ 250. When plotted against variable $y \sqrt{R_{e_{x}}} / x$, the streamwise velocity and temperature profiles taken at different $x$-stations 

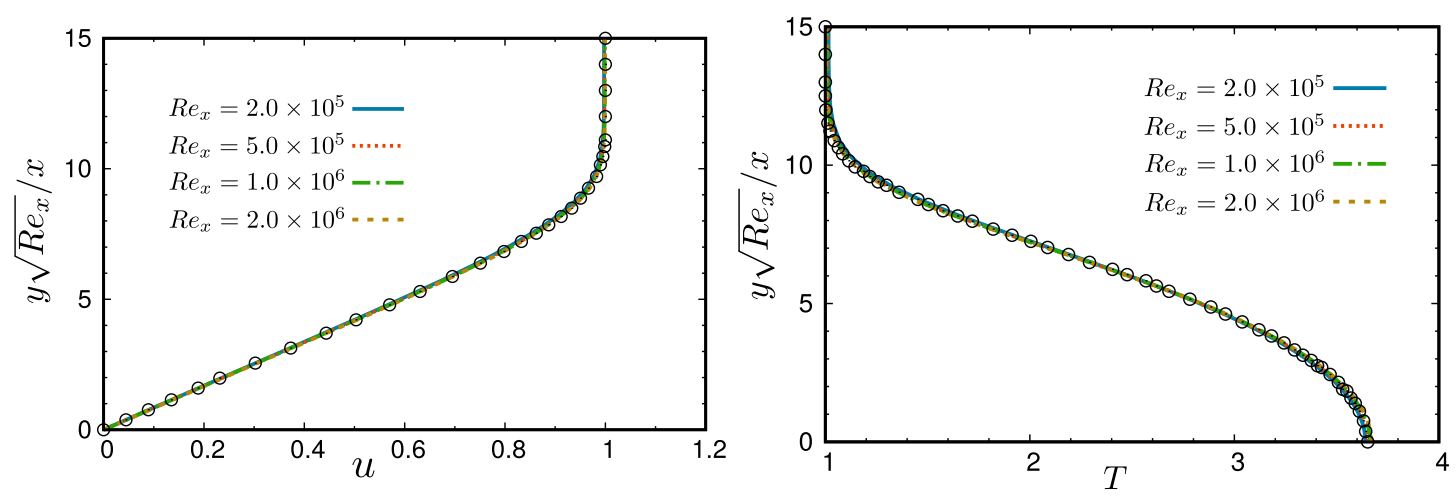

Fig. 2. Supersonic boundary layer flow at $M=4$ : streamwise velocity (left) and temperature (right) profiles against self-similar variable $y \sqrt{R e_{x}} / x$ at different $x$-location. Results from Özgen and Kırcalı [58] are shown in black circle symbols.

collapse, thus recovering the expected self-similar character of this flow (Fig. 2). Furthermore, these profiles are in very good agreement with the results of Özgen and Kırcalı [58] obtained by solving the compressible boundary layer equations.

\subsection{Optimal gain of Blasius boundary layer}

This section provides a validation of the optimal gain solver developed for 3D global perturbations (section 3.3 and 3.5). Comparison to the global results of Monokrousos et al. [34] is proposed. These results were obtained by means of an incompressible solver based on an adjoint formulation, using a time-stepping method and a fringe zone technique. The physical configuration hereby studied is a Blasius boundary layer developing over a flat plate. Reynolds number at inflow (resp. outflow) is set at $R e_{\delta^{*}}=1000$ (resp. $R e_{\delta^{*}}=1836$ ). Using the displacement thickness $\delta_{0}^{*}$ at inflow as the reference length scale, the numerical domain spans over $[0,800] \times[0,30]$.

As the numerical framework of the present paper is for compressible flow, the Mach number is set to $M=0.3$ in order to get a solution close to the incompressible results. First, the baseflow computation is performed using the CFD solver presented in section 3.1. Subsonic boundary conditions are used (see Table 1 ) and $L_{x_{0}}$ is set to $200 \delta_{0}^{*}$. A new numerical domain, which is hereafter used for optimal gain computations, is then obtained by truncating the fields computed from the CFD solver so that they match the domain described in the above paragraph. Optimal gain computations are then performed with the forcing frequency set to $\omega=0$ whereas the wave number $\beta$ varies over $[0,1.2]$. Kinetic energy is used as the norm matrix in equation (12). Note that Monokrousos et al. [34] do not take into account the $1 / 2$ factor in the kinetic energy; therefore, these results are rescaled to match the kinetic energy definition hereby used. The present computation produces slightly lower optimal gain values (around 5\%) compared to the results of Monokrousos et al. [34] (Fig. 3). No compressible effect or mesh influence have been found to account for this discrepancy. It is suggested that this small discrepancy might stem from the fundamentally different approaches used between the reference and the present work. Indeed, Monokrousos et al. [34] computed the time evolution of linearised perturbations on a greater numerical domain $x \in[0,1000]$, where perturbations are damped for $x>800$ by means of a fringe zone technique. The authors thereby suggested to consider

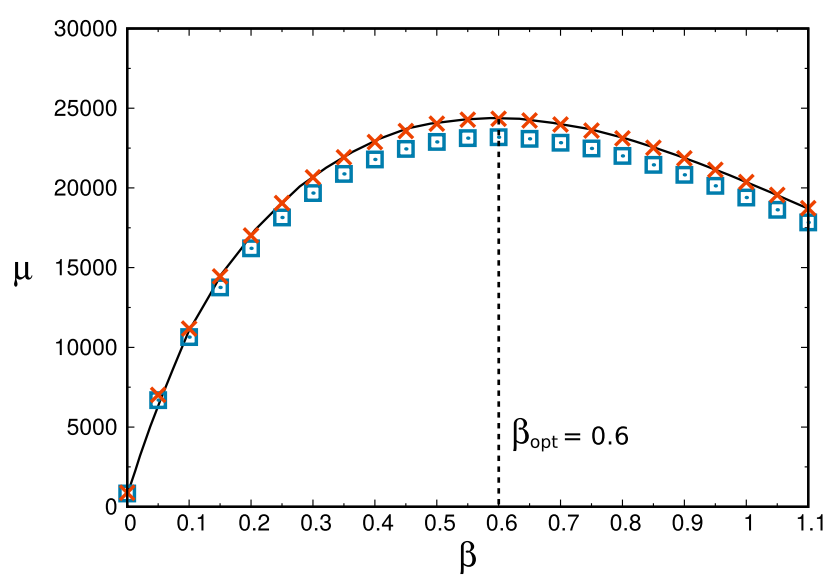

Fig. 3. Optimal gain as a function of the wave number $\beta$ at $\omega=0$ for an incompressible boundary layer. Blue squares: present results, at $M=0.3$. Black line: results of Monokrousos et al. [34] computed for global 3D perturbations in a Blasius boundary layer, using a time-stepping method associated with a fringe zone technique. Red crosses are obtained by rescaling the optimal gain values of the blue squares with those of Monokrousos et al. [34]. 

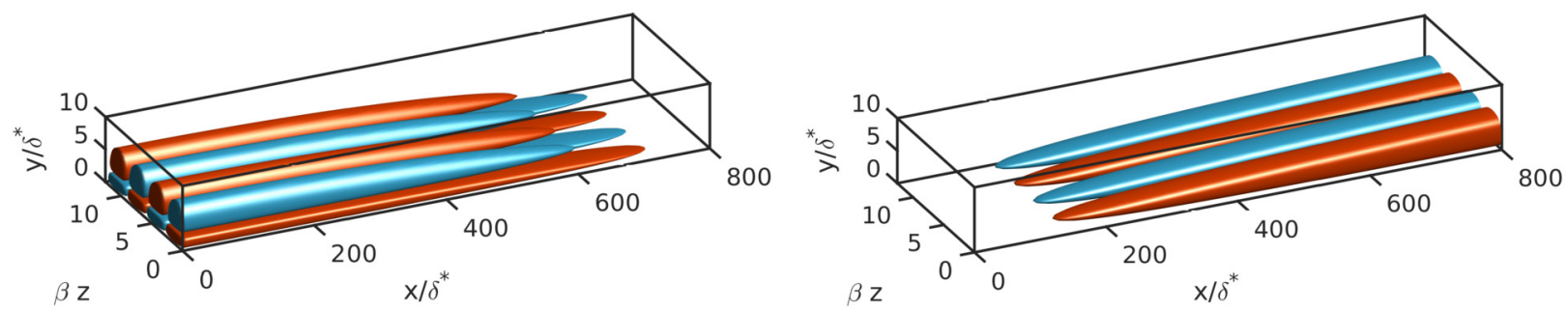

Fig. 4. Real part of optimal forcing component $f_{z}^{\prime}$ (left) and optimal response streamwise velocity $u^{\prime}$ (right) at $\beta=0.6$ and $\omega=0$ for a boundary layer at $M=0.3$. Iso-surfaces at $-10 \%$ and $10 \%$ of the maximum absolute value are shown in red and blue. The numerical domain is truncated in the $y$-direction to ease visualisation.
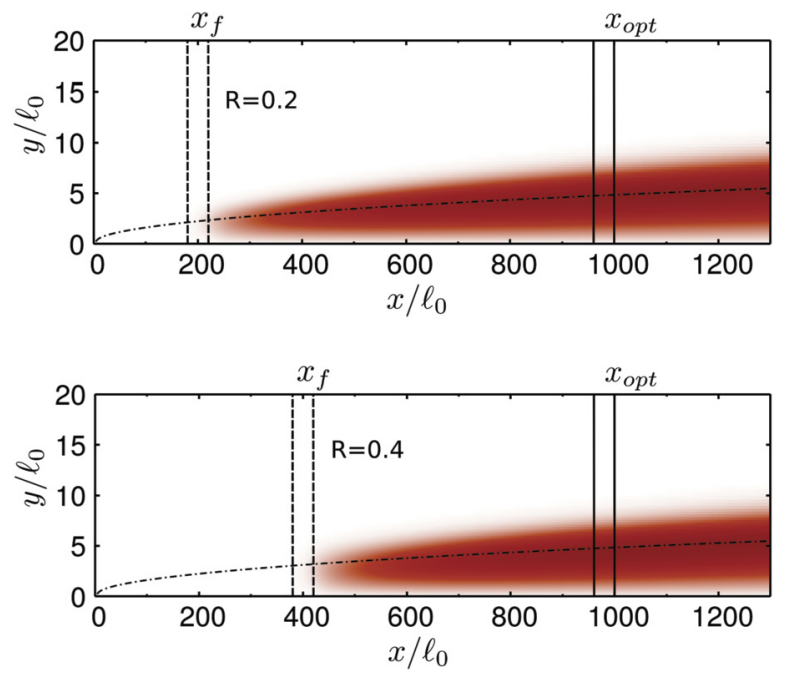

Fig. 5. Real part of $u^{\prime}$ of the optimal response computed for two values of $R=x_{\mathrm{opt}} / x_{\mathrm{f}}$. Here, the forcing field is constrained to be localised between the two vertical dot lines. In both cases, the energy optimisation domain of the response is located between the vertical solid line at $x_{\mathrm{opt}} / \ell_{0}=1000$.

$x \in[0,800]$ as the optimisation domain, which we used in the present computation. However, residual perturbations might persist for $x>800$ and could account for a slightly higher optimal gain value than a computation performed on a numerical domain strictly limited to $x \in[0,800]$. Nevertheless, rescaling the present results allows to observe a perfect agreement between the optimal gain behaviours taken as a function of $\beta$. In particular, the optimal wave number $\beta=0.6$ is retrieved. This agreement is the most significant: indeed, the absolute value of the optimal gain has no physical meaning [35] contrary to the forcing frequency or wave number at which the optimal gain is maximum. The 3D fields depicted in Fig. 4 furthermore support the validity of our computation since the same counter-rotating rolls and streaks topologies as Monokrousos et al. [34] are respectively found as the optimal forcing and response fields associated with the maximum optimal gain value.

\subsection{Optimal gain of a supersonic boundary layer at $M=3$}

To our knowledge, no work dealing with 3D global optimal forcing in compressible flows has been published to this date. In order to validate the method proposed in section 3.3 - which allows to compute 3D optimal global perturbations, a comparison with the "non-global" results from Tumin and Reshotko [21] obtained for a supersonic boundary layer is performed. This method lies on the optimisation of an energetic ratio between perturbation profiles at two different locations, using Chu's energy norm [51]. Hence, this approach is a spatial equivalent of an optimal initial condition computation whose solution is based on the parabolised boundary layer equations by assuming the expected velocity scales of streaks $(u \sim O(1)$ et $v, w \sim O(\varepsilon)[21])$.

In order to compare these results to the global perturbations approach, we suggest to perform a computation in which the forcing field is constrained in a region around a location $x_{\mathrm{f}}$ (Fig. 5) that corresponds to the upstream location used by Tumin and Reshotko [21]. Anywhere outside this region, the forcing field is equal to zero. This is achieved by means of the $\mathbf{P}$ matrix introduced in section 3.5. Moreover, the energy of the response is optimised in a restricted region at location $x_{\mathrm{opt}}$ (Fig. 5) which corresponds to the downstream location used by Tumin and Reshotko [21]. Thereby, the relation between our global approach and the "non-global" approach used as a reference is the following: the computed forcing fields at $x_{\mathrm{f}}$ plays the role of the optimal condition, the response fields at $x_{\mathrm{opt}}$ is the perturbation that grows downstream and the optimal gain mimics the energetic ratio. 

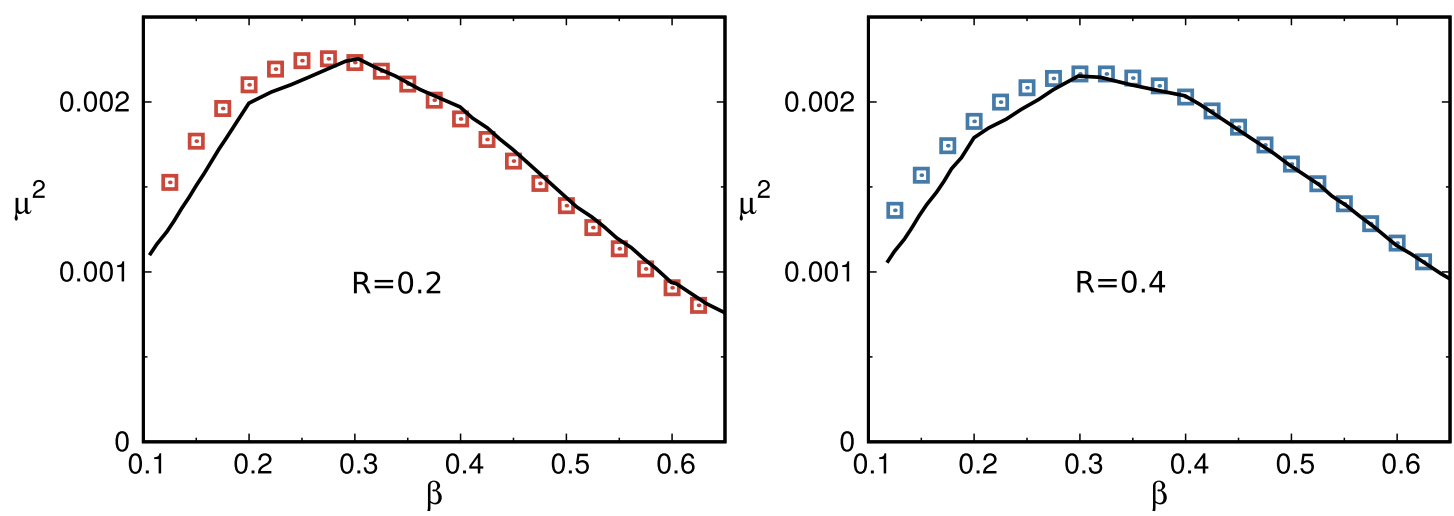

Fig. 6. Optimal gain computed at $\omega=0$ with constrained forcing and response fields for the compressible boundary layer at $M=3$, for two values of $R=x_{\mathrm{opt}} / x_{\mathrm{f}}$. Black line: results from Tumin and Reshotko [21]. Reference length scale is $\ell_{0}=\sqrt{\eta_{\infty} x_{\mathrm{opt}} / \rho_{\infty} u_{\infty}}$. Square symbols: present results.

Table 4

Physical and numerical parameters of the baseflow.

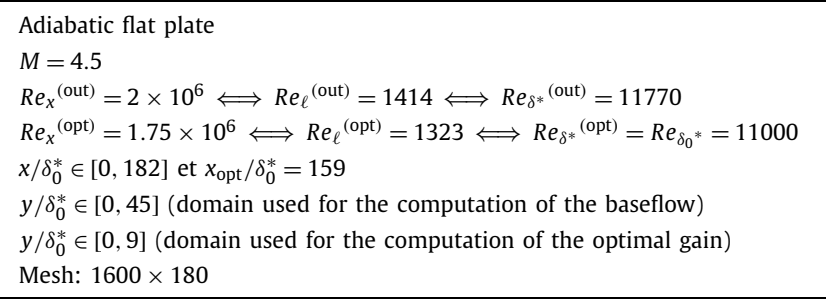

The baseflow computation is performed with the Mach number set at $M=3$ and the Reynolds number, based on the Blasius length scale $\ell=\sqrt{\eta_{\infty} X / \rho_{\infty} u_{\infty}}$, set at $R e_{\ell}=1140$ at outflow. The value of $x_{\text {opt }}$ is set at the abscissa corresponding to $R e_{\ell}=1000$ and the Blasius length at this specific abscissa, $\ell_{0}=\sqrt{\eta_{\infty} X_{\mathrm{opt}} / \rho_{\infty} u_{\infty}}$, will be used as the reference length scale. Two different values of $x_{\mathrm{f}}$ will successively be considered such that the ratio $R=x_{\mathrm{f}} / x_{\mathrm{opt}}$ is equal to 0.2 and 0.4 . The numerical domain spans over $x / \ell_{0} \in[0,1300]$ and $y / \ell_{0} \in[0,100]$. The regions over which the forcing and response fields are constrained span over $\Delta x / \ell_{0}=40$ in the streamwise direction but are not restricted in the normal direction. Results of optimal gain computations are compared to those of Tumin and Reshotko [21] by renormalising the optimal gain. Indeed, the definitions of these two quantities are different and their absolute value cannot be directly compared. A very good agreement is observed for the two ratios $R$ considered (Fig. 6). The optimal response fields associated with the maximum optimal gain shows that the growth of streaks starts from the forcing location (Fig. 5). The convectively unstable nature of these structures is observed as their growth goes on downstream from the region where they are forced.

\section{Optimal forcing and response of the supersonic boundary layer at $M=4.5$}

\subsection{Baseflow}

The baseflow used for optimal gain computations (section 5.2) is presented in this section. A boundary layer developing over an adiabatic flat plate is considered at $M=4.5$, at which local stability analysis show that Mack mode reaches its maximum growth rate [59]. Physical and numerical parameters are reported in Table 4 where the Reynolds number is computed according to different reference length scales. Local stability studies usually take Blasius length $\ell=\sqrt{\eta_{\infty} x / \rho_{\infty} u_{\infty}}$ as reference, which is associated with the plate abscissa $x$ according to $R e_{\ell}=\sqrt{R e_{x}}$. Here, compressible displacement thickness $\delta^{*}(x)$ will be considered. As expected, the relation between $R e_{\ell}$ and $R e_{\delta^{*}}$ obtained from our numerical computation is observed to be linear as a consequence of self-similarity (Fig. 7). The corresponding local Mach number field is depicted in Fig. 8. Note that the domain over which Chu's disturbances energy is integrated during optimal gain computation is defined over $x<x_{\text {opt }}$, where $x_{\text {opt }}$ is the abscissa where $R e_{\delta^{*}}=11000$. The compressible displacement boundary $\delta_{0}^{*}$ at this abscissa will be used as the reference length. It should be pointed out that this length appears both in the non-dimensional forcing frequency $\omega$ and wave number $\beta$. Finally, the actual baseflow used for optimal gain computation is truncated in the $y$-direction for computational savings. It is shown in Appendix D that the results are independent from this choice of domain. 


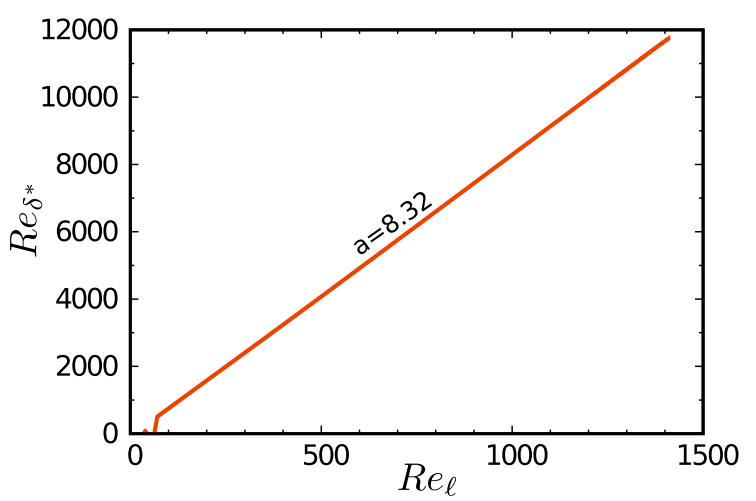

Fig. 7. Computed Reynolds number $R e_{\delta^{*}}$ based on the compressible displacement thickness as a function of the Reynolds number $R e_{\ell}$ based on Blasius length scale. $a$ is the slope of the linear curve, obtained by linear regression $\left(r^{2}>0.99\right)$.

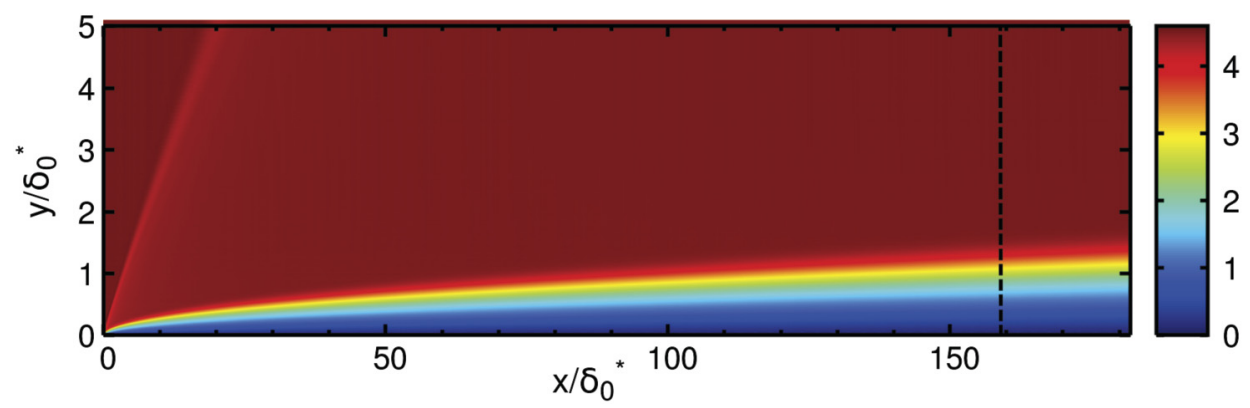

Fig. 8. Local Mach number field of the baseflow at $M=4.5$. Optimisation domain used for optimal gain computation is located upstream from the vertical dotted line. Note that the numerical domain is truncated in the $y$-direction to ease the visualisation.

\subsection{Optimal gain}

Results from optimal gain computations for 3D perturbations developing over the baseflow presented in section 5.1 are shown in Fig. 9. Mesh convergence and computational cost are given in Appendix D and Appendix E. In these calculations, the forcing field is not constrained to be localised in any zones of the flow. Three regions of maximum gain can be identified in the $\omega-\beta$ space, where the flow is therefore especially receptive to an external forcing. Optimal forcing frequency and wave number associated with these three regions are shown in Table 5. As the forcing frequency goes to zero, an optimal wave number is found to favour the non-modal growth of streaks. At medium frequency, a peak of optimal gain is detected for non-zero wave numbers (approximately half the value of the streaks one), which implies that the associated perturbation has an oblique wave structure. This is the first mode instability of the compressible boundary layer [11]. At high frequency, a maximum of optimal gain is reached for zero wave number and pertains to the growth of the second mode (Mack mode). Spatial structures of each optimal forcing and response corresponding to the three optimal gain maximums will be analysed in section 5.3.

The interpretation of the values reached by the gain peaks must be done cautiously. Indeed, as pointed out in section 3.5, the absolute value of the optimal gain has no physical meaning. Relative values can however be compared in order to assess the efficiency of the different receptivity mechanisms. Here, the maximum optimal gain value is associated with the first mode instability whereas the streaks has a lower value within the same order of magnitude. The second mode has an optimal gain one order of magnitude lower than the first mode. Note that this is not in contradiction with the fact that the second mode growth rate is twice higher than the first mode one obtained from local stability computations [11]. Indeed, the optimal gain is a global quantity that accounts for the energy growth of perturbations over a given physical domain. Thus it depends on both the growth rate of the instability and the length over which it grows, that is the width of its neutral stability curve at a given frequency. The analysis of the energy growth profiles plotted in section 5.4 will shed more light on this matter.

\subsection{Analysis of the optimal forcing and response}

Spatial structures of the optimal forcing and response associated with the three regions of maximal gain are examined in this section. At low frequency (Fig. 10), the lift-up mechanism is recovered. The optimal forcing is made of streamwise counter-rotating rolls that initiate the transport of streamwise momentum of the baseflow by the perturbation. Streaks of 


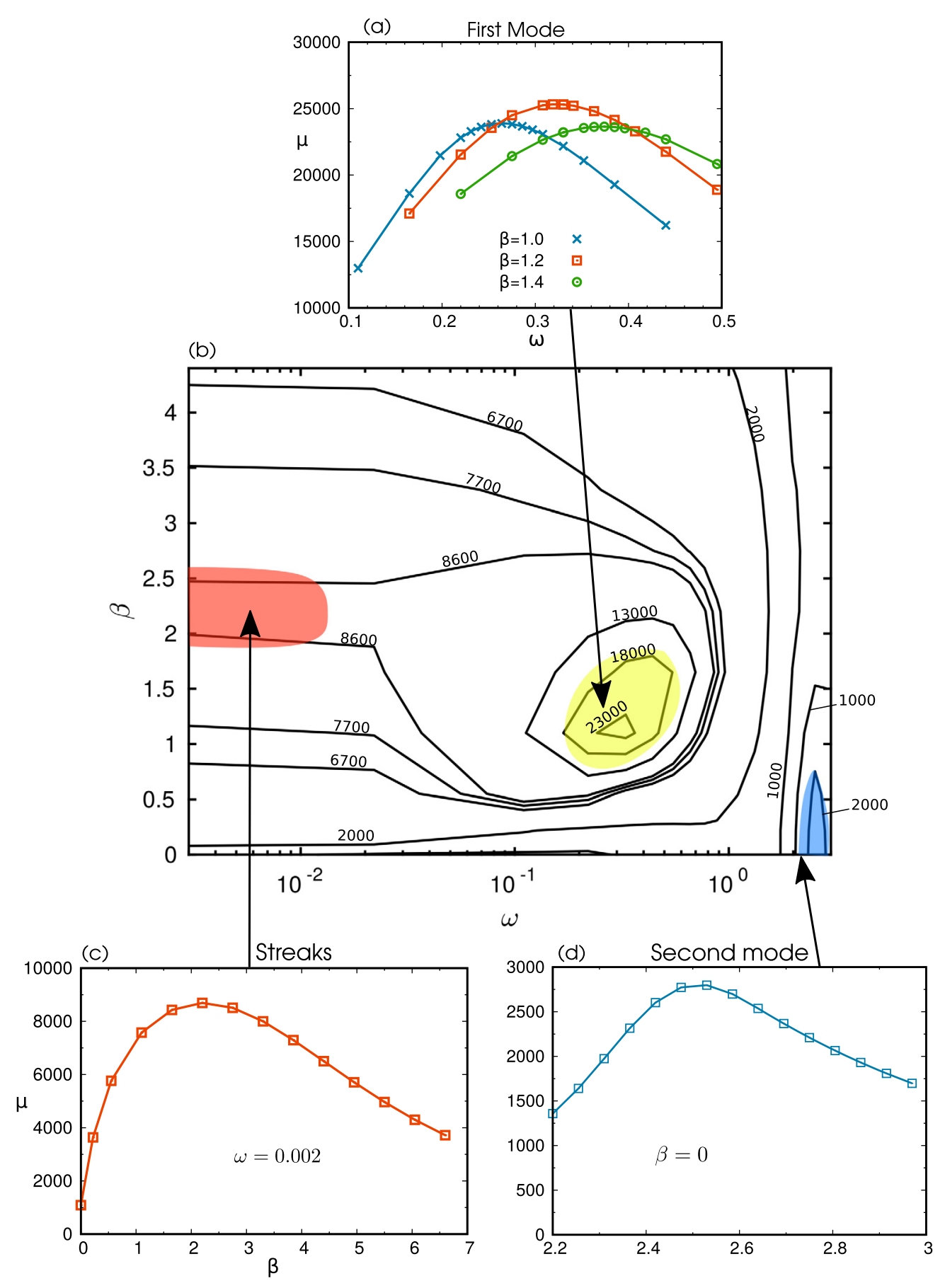

Fig. 9. (b) Optimal gain of the compressible boundary layer at $M=4.5$ for 3D perturbations is plotted in the $\omega-\beta$ space. Three regions of locally maximum gain are detected and are associated with three linear instabilities. (a) Optimal gain associated with first mode instability is plotted against $\omega$ at $\beta=1.0$, $\beta=1.2$ and $\beta=1.4$. (c) Optimal gain associated with streaks is plotted against $\beta$ at $\omega=0.002$. (d) Optimal gain associated with second mode instability is plotted against $\omega$ at $\beta=0$.

Table 5

Optimal forcing frequency and wave number for each instability.

\begin{tabular}{lll}
\hline Instability & $\omega_{\text {opt }}$ & $\beta_{\text {opt }}$ \\
\hline Streaks & $\rightarrow 0$ & 2.2 \\
First mode & 0.32 & 1.2 \\
Second mode & 2.5 & 0 \\
\hline
\end{tabular}



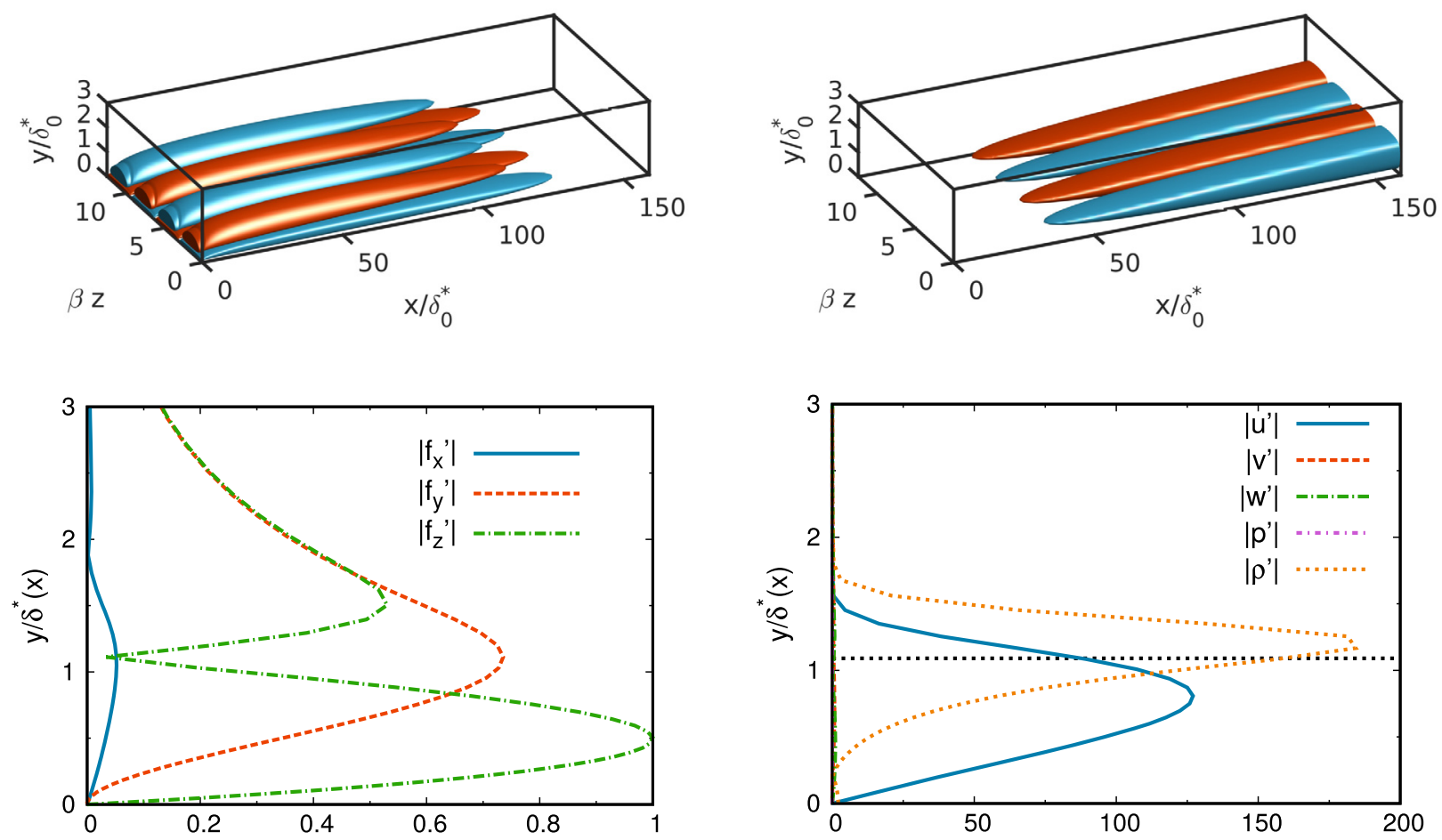

Fig. 10. Optimal forcing (left) and response (right) at $\omega=0.002$ and $\beta=2.2$. Top: Iso-surfaces at $10 \%$ of $\tilde{f}_{z}$ (left) and $\widetilde{u}$ (right). Note that the spanwise axis is normalised using the wave number of the perturbations. Bottom: Profiles at the streamwise location corresponding to the maximum forcing density (left) and Chu's energy density (right). Profiles at $x / \delta_{0}^{*}=35$ (left) and $x / \delta_{0}^{*}=159$ (right). Forcing components are normalised by the maximum value of $\widetilde{f}_{z}$. Black dotted line indicates the generalised inflection point.

high streamwise velocity, spanning in the streamwise direction, are thus generated which correspond to the local analysis results predicting a zero streamwise wave number [60]. These fields are actually similar to those obtained in an incompressible boundary layer [34], showing that the lift-up effect can be generalised to compressible flow [19]. Note, however, that a peak of density appears in the response profile above the streamwise velocity peak and close to the generalised inflection point $y_{i}$ of the baseflow, which is defined for each streamwise station as $\partial / \partial y[\rho \partial \bar{u} / \partial y]\left(y_{i}\right)=0$. This feature has theoretical grounds in a local, modal instability framework [59] (it will indeed be observed in the optimal responses associated with the first and second modes, as described further in this section). It is here noted that the non-modal growth of streaks also shares this behaviour.

At medium frequency, optimal forcing and response fields appear as oblique waves (Fig. 11). Assuming a wavelike structure of the perturbation fields $\widetilde{\mathbf{q}}(x, y)=\widehat{\mathbf{q}}(y) e^{i \alpha x}=\widehat{\mathbf{q}}(y) e^{i \alpha_{r} x} e^{-\alpha_{i} x}$, the streamwise wave number $\alpha_{r}$ can be computed as

$$
\alpha_{r}=\Re\left(\frac{1}{i \widetilde{\mathbf{q}}} \frac{\partial \widetilde{\mathbf{q}}}{\partial x}\right)
$$

where $\Re$ stands for the real part of the complex quantity. In practice, the streamwise velocity profile $\widetilde{u}(x)$ at $y=1$ is used in equation (50). The wave angle compared to the baseflow direction can then be computed according to

$$
\psi=\tan ^{-1}\left(\frac{\beta}{\alpha_{r}}\right)
$$

Here, the angle of the optimal response is found to be equal to $72^{\circ}$ which can be compared to the angle of $60^{\circ}$ of the most unstable first mode obtained from a local stability analysis [11]. Since the optimal response is not on the one hand strictly modal in nature and on the other hand is based on a global and not a global analysis, there is no reason to find the $60^{\circ}$ value of a first mode wave computed with a local approach (a comparison with results from an $e^{N}$ method would here be interesting, but is beyond the scope of the present study). Nevertheless, both approaches do show that growth of the first mode is stronger as an oblique wave. From Fig. 11, it is observed that the forcing fields tend to be localised in the upstream region of the numerical domain whereas the response grows downstream as a consequence of streamwise non-normality [37]. Moreover, the iso-surfaces of the forcing field are tilted upstream which suggests the action of the non-modal Orr mechanism. The examination of the disturbances profile (Fig. 11) shows that transverse forcing is the most efficient and that it mainly generates a streamwise velocity. Compared to incompressible Tollmien-Schlichting waves, the velocity profile appears further away from the wall, close to the generalised inflection point which pertains to the prevalent inviscid mechanism of the first mode instability as Mach number increases. 

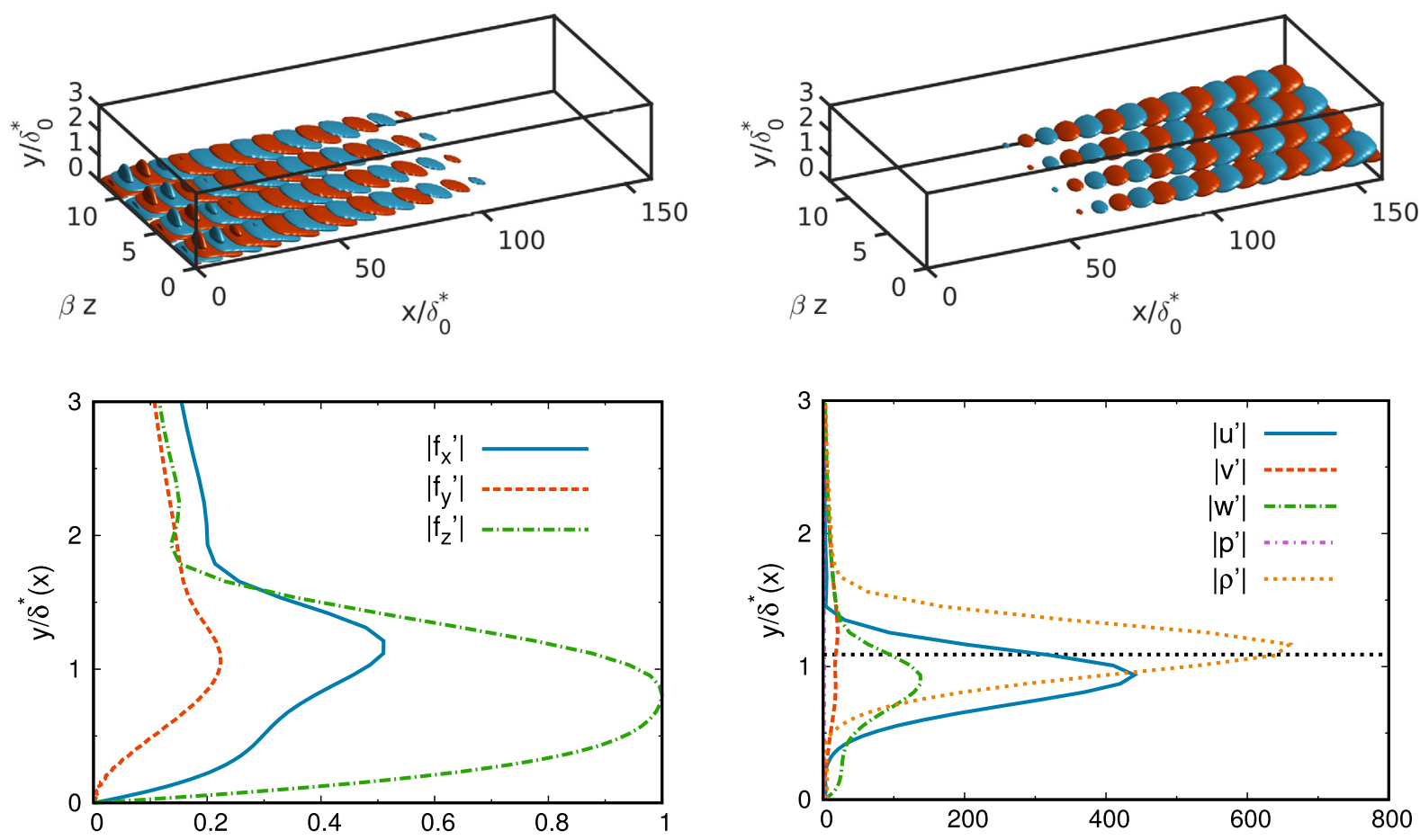

Fig. 11. Optimal forcing and response at $\omega=0.32$ and $\beta=1.2$. See caption in Fig. 10. Bottom: Profiles at $x / \delta_{0}^{*}=12$ (left) and $x / \delta_{0}^{*}=159$ (right).

Optimal forcing and response at high frequency are now examined. From the wave number computed following (50), the phase velocity $c_{\varphi}$ and subsequently the relative Mach number field $\widehat{M}[8,11]$ of the optimal response can be obtained, the latter being defined as

$$
\widehat{M}=\frac{\bar{u}-c_{\varphi}}{\bar{c}}
$$

where $\bar{c}$ is the speed of sound computed from the baseflow. A supersonic region $\widehat{M}>1$ is detected close to the wall (Fig. 12) which is, according to Mack [11], a condition for additional unstable modes to exist. In this region, profiles show that each physical quantity of the optimal response reaches a maximum. The optimal forcing is however not very active in this part of the flow. Instead, it tends to be localised near the generalised inflection point where, in addition, density reaches another peak. Hence, two distinct mechanisms seem to coexist. On the one hand, the growth of hydrodynamical and thermodynamical perturbations inside the supersonic region seem to purely pertain to the second mode instability. On the other hand, the thermodynamical perturbations are also amplified along the generalised inflection point. Note that similar density peaks were observed for streak and first mode optimal responses. This property, that seems shared by these three different compressible instabilities, can also be observed in the studies of Hanifi et al. [19] and Erlebacher and Hussaini [61].

\subsection{Energy growth}

In this section, the energy growth of the optimal responses are examined in order to further characterised the development of the three convective instabilities previously exhibited. In order to assess these behaviours, energy densities can be defined at each streamwise station [35]. For example, the energy density associated with the kinetic energy is taken as $\mathrm{d}_{\mathrm{K}}(x)=\int_{0}^{y_{\max }} \bar{\rho}|\widetilde{\mathbf{u}}|^{2} \mathrm{~d} y$. The Chu's energy density is constructed in a similar way and is referred to as $\mathrm{d}_{\text {Chu }}(x)$. The forcing density is also defined as $\mathrm{d}_{\mathrm{F}}(x)=\int_{0}^{y_{\max }}|\widetilde{\mathbf{f}}|^{2} \mathrm{~d} y$ and the streamwise evolution of these quantities are plotted in Fig. 13. It is observed that the maximum forcing density associated with the first mode response is located far upstream from the one of the second mode. The energy growth of the first mode also starts more upstream than the second mode and continues until the end of the optimisation domain whereas the growth of the second mode stops slightly before the downstream boundary. This allows to shed light on why the optimal gain associated with the first mode is higher than that of the second mode. Indeed, even if its amplification is slower, it spans over a longer length. These observations are consistent with the neutral stability curve obtained by Malik and Balakumar [62], where it can be seen that, in the frequency range studied here, the lower (respectively upper) branch of the first mode is found at a lower (respectively higher) Reynolds number than the second mode. 

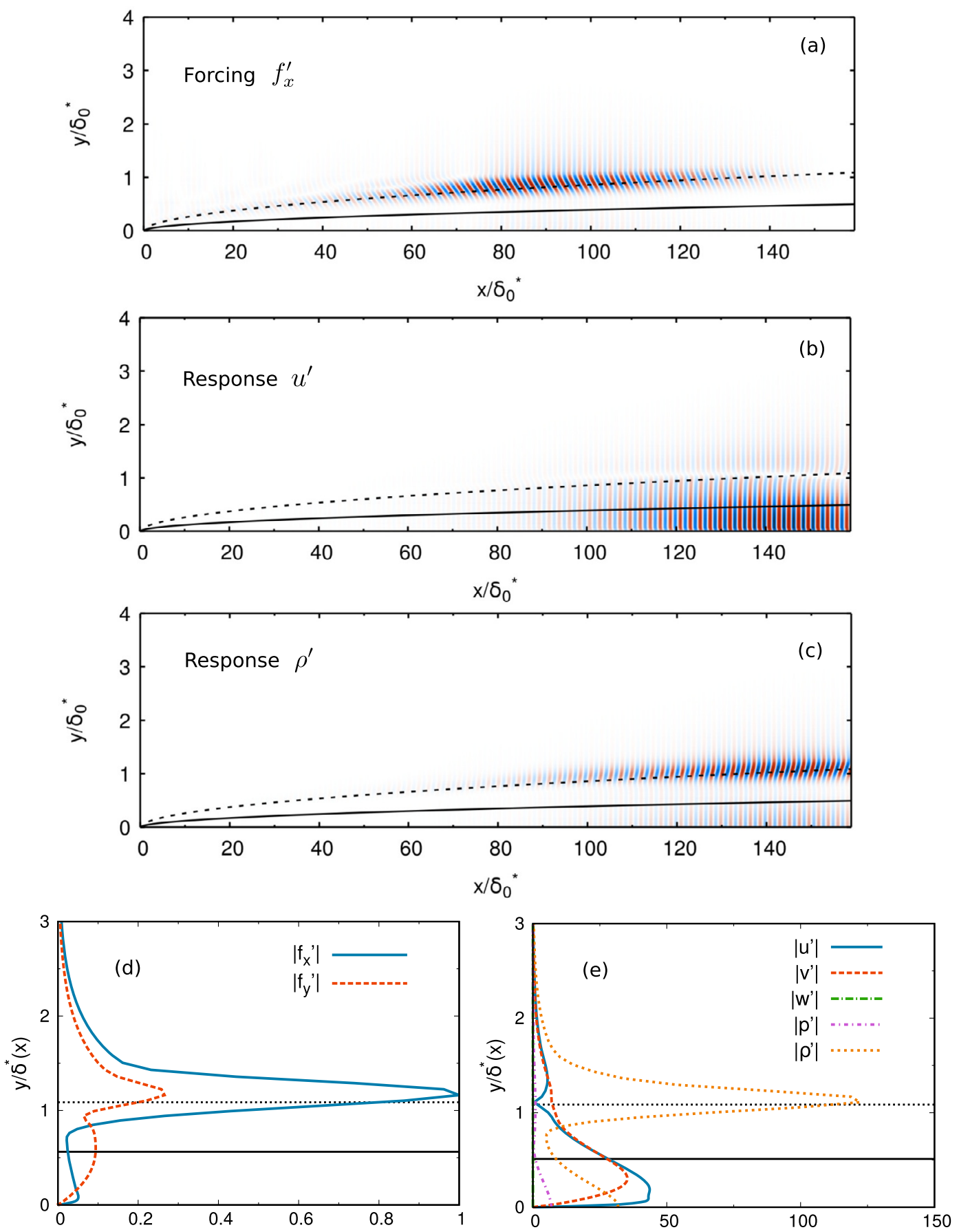

Fig. 12. Streamwise component of the optimal forcing (a) and streamwise velocity (b) and density (c) of the optimal response at $\omega=2.5$ and $\beta=0$. (d) and (e): Profiles at $x / \delta_{0}^{*}=90$ (left) and $x / \delta_{0}^{*}=148$ (right). See caption in Fig. 10 . Here, the forcing components are normalised by the maximum value of $\tilde{f}_{x}$. Black solid line indicates the ordinate where $\widehat{M}=-1$ (supersonic region is located below this line).

Because the streaks result from a purely non-modal instability, interpreting its energy growth cannot be done through neutral curve considerations. However, it is observed that the streaks growth occurs over a larger length than the first and second mode. Besides, the forcing density profile is more spread than that of the first and second modes, the latter being more localised around a particular streamwise location. Using the terminology described by Sipp et al. [31], these observations can be interpreted in terms of convective-type and component-type non-normalities, which are responsible for the growth of these different instabilities. The convective-type non-normality is at play for both the first and second modes as the support of the forcing and response fields are clearly separated in the streamwise direction, respectively upstream 

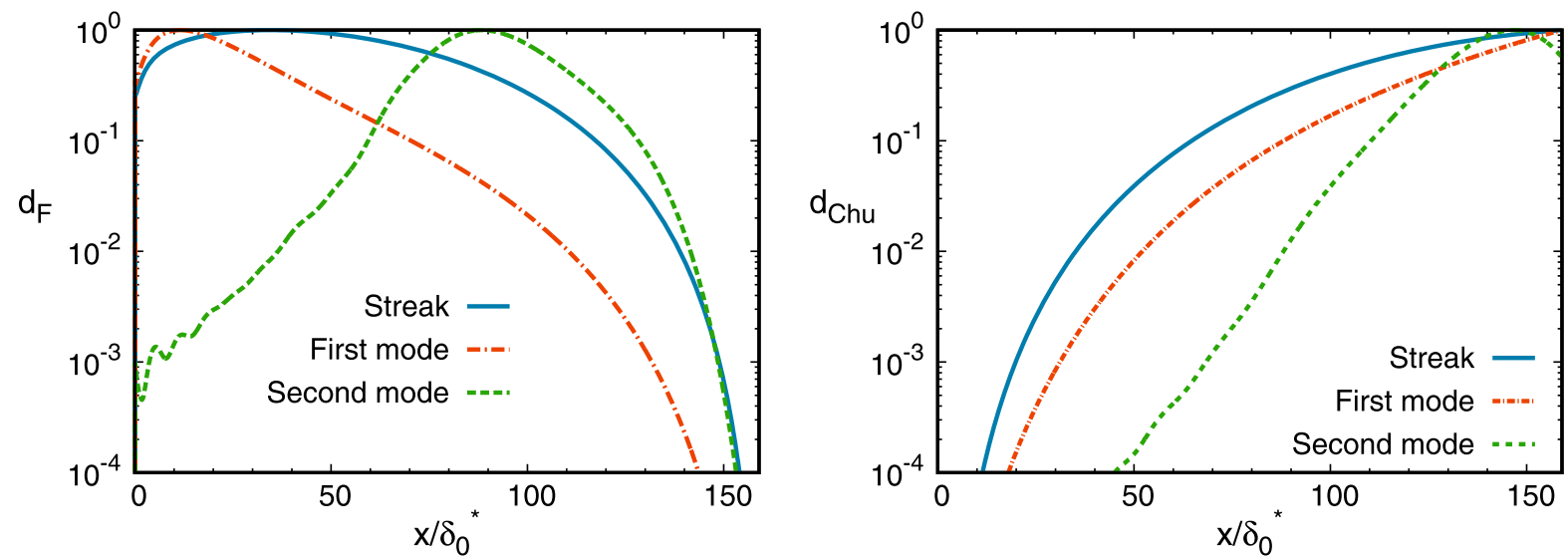

Fig. 13. Forcing density (left) and Chu's energy density (right) of optimal forcing and response associated with optimal gain maxima (see Table 5).
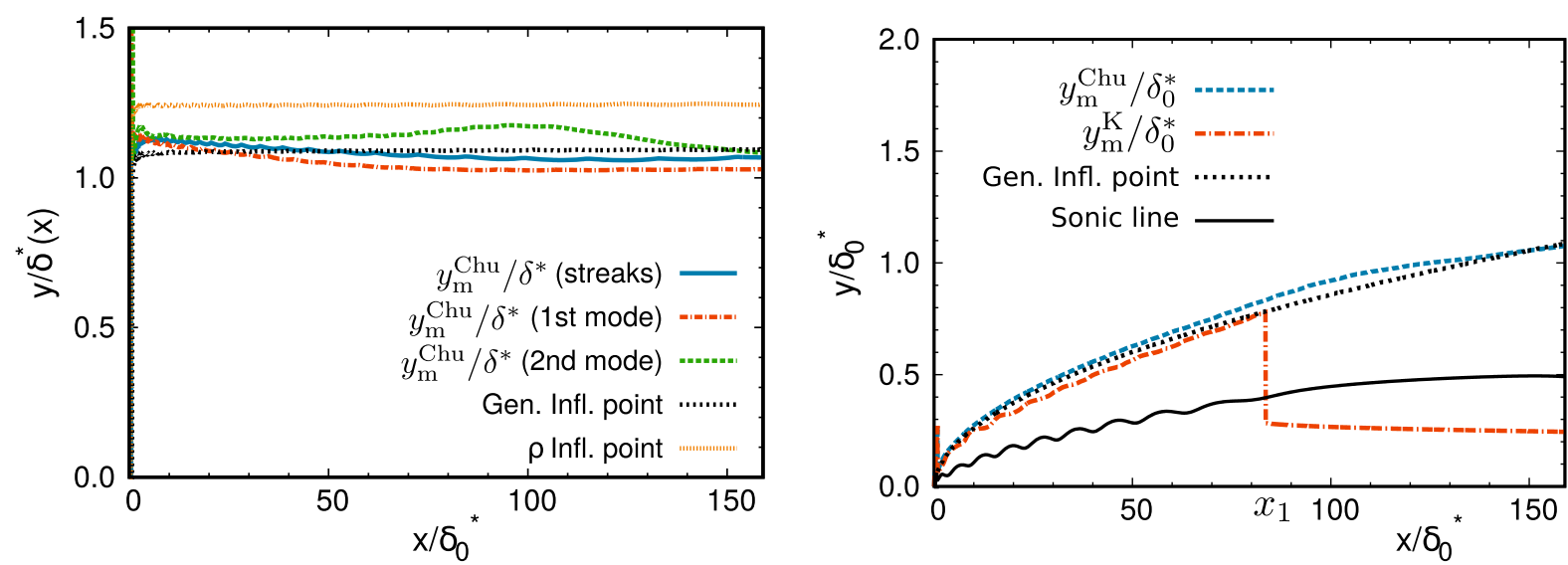

Fig. 14. Left: Streamwise evolution of $y_{\mathrm{m}}^{\text {Chu }}$ for each optimal response associated with optimal gain maximums (see Table 5). $y_{\mathrm{m}}^{\text {Chu }}$ is normalised by the value of $\delta^{*}(x)$ at each streamwise station. Right: $y_{\mathrm{m}}^{\mathrm{Chu}}$ and $y_{\mathrm{m}}^{\mathrm{K}}$ (of the second mode only) normalised by $\delta_{0}^{*}$ at $x=x_{\mathrm{opt}}$. The black and orange dotted lines respectively indicate the location of the generalised inflection point and the inflection point of the density profile. The location where $\widehat{M}=-1$ is shown by a black solid line.

and downstream [37]. In the case of the streaks growth, the component-type non-normality is active as it is related to the lift-up mechanism. This mechanism is local in the sense that, at each streamwise location, the growth of the response takes advantage of the transport in the normal direction of the baseflow momentum by the perturbations. As such, a local support of the forcing field over a large portion of the streaks growth domain appears to be more efficient.

To further characterise the energy growth of the optimal responses, the ordinate at which the density energy reaches its maximum is computed at each streamwise station. It is formally defined for Chu's energy and kinetic energy as

$$
\begin{aligned}
y_{\mathrm{m}}^{\mathrm{Chu}}(x) & =\underset{y}{\arg \max }\left[\bar{\rho}|\widetilde{\mathbf{u}}|^{2}+\frac{\bar{T}}{\bar{\rho} \gamma M^{2}} \widetilde{\rho}^{2}+\frac{\bar{\rho}}{(\gamma-1) \gamma M^{2} \bar{T}} \widetilde{T}^{2}\right] \\
y_{\mathrm{m}}^{\mathrm{K}}(x) & =\underset{y}{\arg \max }\left[\bar{\rho}|\widetilde{\mathbf{u}}|^{2}\right]
\end{aligned}
$$

When normalised by the local displacement thickness $\delta^{*}(x)$, it is found that $y_{\mathrm{m}}^{\text {Chu }}$ is constant along the plate (Fig. 14). Hence, the Chu's energy profile grows as $\sqrt{x}$, which can be seen as a property inherited from the baseflow (see section 5.1 ). Moreover, $y_{\mathrm{m}}^{\mathrm{Chu}}$ is localised close to the generalised inflection point. The inflection point of the density profile is found to be less relevant to predict the peak of energy as it is localised slightly higher. However, these observations hide the growth of the second mode perturbation in the relative Mach number supersonic region close to the wall (Fig. 12). This is revealed in Fig. 14 by plotting $y_{\mathrm{m}}^{\mathrm{K}}$ which is normalised by the non-local displacement thickness $\delta_{0}^{*}$. Both $y_{\mathrm{m}}^{\mathrm{K}}$ and $y_{\mathrm{m}}^{\text {Chu }}$ evolve along the general inflection point until the abscissa $x_{1} / \delta_{0}^{*}=83$. But for $x>x_{1}, y_{\mathrm{m}}^{\mathrm{K}}$ is located closer to the wall inside the supersonic region and does not scale as $\sqrt{x}$ any more. This steep modification of growth indicates the start of the second mode instability as $x_{1}$ also corresponds to the maximum of forcing density (Fig. 13). Indeed, this observation is in agreement with the work of Sipp and Marquet [35] who observed, for a Blasius boundary layer, that the location of 
the forcing density coincides with the location of the lower branch of a convective instability. Finally, although not shown here, note that $y_{\mathrm{m}}^{\mathrm{K}}$ still grows as $\sqrt{x}$ for streaks and first mode instabilities. In this case, it is observed that $y_{\mathrm{m}}^{\mathrm{K}}<y_{\mathrm{m}}^{\mathrm{Chu}}$ since the energy peak, which is located further away from the wall than velocities peaks, is not taken into account in kinetic energy.

\section{Conclusion}

A numerical method allowing to compute the Jacobian matrix associated with 3D global perturbations developing over a $2 \mathrm{D}$ baseflow has been proposed. This method is an extension of the discretised-then-linearised procedure introduced by Mettot et al. [1] for 2D global perturbations which is particularly suited for compressible flows. Because a Fourier expansion is performed in the transverse direction, modifications of the $2 \mathrm{D}$ method have been required: the Jacobian matrix has been separated into three conveniently computable matrices. The numerical implementation of the 3D solver from an available 2D solver is straightforward and the increase of computational cost is kept affordable. In order to study convectively unstable compressible flows, optimal gain computations have then been performed to compute 3D global optimal forcing and response. This approach is based on the computation of the largest singular value of the global resolvent operator (built from the Jacobian matrix) and takes into account both modal and non-modal effects (resonance and pseudo-resonance) involved in the growth of perturbations subject to an external forcing. The validation of the numerical method has first been achieved for a Blasius boundary layer, for which global results were available from an incompressible solver [34]. Afterwards, validation against non-global results of a supersonic boundary layer [21] has been performed given that no global results were so far known.

To demonstrate the potential of the numerical method, a detailed study of the receptivity of the supersonic boundary layer at $M=4.5$ and $R e_{\delta^{*}}=11000$ has finally been presented. Optimal gain computations as a function of the forcing frequency $\omega$ and the transverse wave number $\beta$ has revealed three regions of locally maximum optimal gain value. They are associated with the growth of compressible streaks $(\omega \rightarrow 0, \beta=2.2)$, first mode instability as an oblique wave $(\omega=0.32$, $\beta=1.2)$ and second mode instability $(\omega=2.5, \beta=0)$. The generalised inflection point has been shown to play a role in the growth of thermodynamical perturbations and Chu's energy growth profiles along the flat plate have been found to evolve as $\sqrt{x}$. These findings have been moderated regarding the second mode instability as the profiles of hydrodynamical perturbations have been observed to grow independently of $x$ inside the supersonic relative Mach number region close to the wall.

As the numerical method does not require any assumptions on the non-parallel nature of the baseflow, future work may tackle more complex flows. For example, 3D receptivity of the shock wave/boundary interaction could be further examined [43]. Exhibiting the main features of the 3D dynamics of industrial flows, involving complex geometries, could also be achieved while keeping affordable computational costs. Asymmetrical flows could moreover be studied providing that the mathematical framework is extended to cylindrical coordinates. This would especially be relevant to analyse the acoustic radiation associated with the growth of wave packets in turbulent jet flows [63]. Finally, application of the numerical method to the control of compressible flows is a promising perspective. 3D perturbations, as we have shown for the supersonic boundary layer, can reach larger optimal gain values than 2D perturbations and can potentially represent the most dangerous instabilities that one would want to control. From the construction of the Jacobian matrix of 3D perturbations presented in this paper, and by following the adjoint and Hessian based method proposed by Mettot et al. [1] in a discrete framework, passive control strategies of 3D convective instabilities in compressible flows could be implemented.

\section{Declaration of competing interest}

The authors declare that they have no known competing financial interests or personal relationships that could have appeared to influence the work reported in this paper.

\section{Acknowledgements}

This work was supported by the French government (three-year doctoral contract).

\section{Appendix A. Numerical fluxes used for the 3D perturbations Jacobian matrix}

The complete expressions of the flux separation performed in section 3.3 for the computation of the Jacobian matrix for 3D perturbations are given hereafter. Considering the Navier-Stokes fluxes $\mathbf{F}, \mathbf{G}$ and $\mathbf{H}$, the strategy lies on isolating the terms containing transverse derivatives $\partial / \partial z$ into vectors $\mathbf{F}_{v z}, \mathbf{G}_{v z}$ and $\mathbf{H}_{v z}$ and writing the remaining terms into vectors $\mathbf{F}^{\prime}$, $\mathbf{G}^{\prime}$ and $\mathbf{H}^{\prime}$. 
$\mathbf{F}=\mathbf{F}^{\prime}-\mathbf{F}_{v z}$

$$
\begin{aligned}
& \mathbf{F}=\left(\begin{array}{c}
\rho u \\
\rho u^{2}+p-\frac{1}{R e} \tau_{x x} \\
\rho u v-\frac{1}{R e} \tau_{x y} \\
\rho u w-\frac{1}{R e} \tau_{x z} \\
u(\rho E+p)-\frac{1}{\operatorname{Re}}\left[u \tau_{x x}+v \tau_{x y}+w \tau_{x z}\right]-\frac{\lambda}{\operatorname{PrRe}(\gamma-1) M_{\infty}^{2}} \frac{\partial T}{\partial x}
\end{array}\right) \\
& \mathbf{F}^{\prime}=\left(\begin{array}{c}
\rho u \\
\rho u^{2}+p-\frac{\eta}{R e}\left(\frac{4}{3} \frac{\partial u}{\partial x}-\frac{2}{3} \frac{\partial v}{\partial y}\right) \\
\rho u v-\frac{\eta}{R e}\left(\frac{\partial u}{\partial y}+\frac{\partial v}{\partial x}\right) \\
\rho u w-\frac{\eta}{R e} \frac{\partial w}{\partial x} \\
u(\rho E+p)-\frac{\eta}{R e}\left[u\left(\frac{4}{3} \frac{\partial u}{\partial x}-\frac{2}{3} \frac{\partial v}{\partial y}\right)+v \frac{\eta}{R e}\left(\frac{\partial u}{\partial y}+\frac{\partial v}{\partial x}\right)+w \frac{\eta}{R e} \frac{\partial w}{\partial x}\right]-\frac{\lambda}{\operatorname{PrRe}(\gamma-1) M_{\infty}^{2}} \frac{\partial T}{\partial x}
\end{array}\right) \\
& \mathbf{F}_{v z}=\left(\begin{array}{c}
0 \\
-\frac{2}{3} \frac{\eta}{R e} \frac{\partial w}{\partial z} \\
0 \\
\frac{\eta}{R e} \frac{\partial u}{\partial z} \\
\frac{\eta}{R e}\left[-u \frac{2}{3} \frac{\partial w}{\partial z}+w \frac{\partial u}{\partial z}\right]
\end{array}\right)
\end{aligned}
$$

$\mathbf{G}=\mathbf{G}^{\prime}-\mathbf{G}_{v z}$

$$
\begin{aligned}
& \mathbf{G}=\left(\begin{array}{c}
\rho v \\
\rho u v-\frac{1}{R e} \tau_{y x} \\
\rho v^{2}+p-\frac{1}{R e} \tau_{y y} \\
\left.\rho v w-\frac{1}{\operatorname{Re}} \tau_{y z}\right]-\frac{\lambda}{\operatorname{PrRe}(\gamma-1) M_{\infty}^{2}} \frac{\partial T}{\partial y}
\end{array}\right) \\
& \mathbf{G}^{\prime}=\left(\begin{array}{c}
\rho v \\
\rho u v-\frac{\eta}{R e}\left(\frac{\partial u}{\partial y}+\frac{\partial v}{\partial x}\right) \\
\rho v^{2}+p-\frac{\eta}{R e}\left(\frac{4}{3} \frac{\partial v}{\partial y}-\frac{2}{3} \frac{\partial u}{\partial x}\right) \\
\rho v w-\frac{\eta}{R e} \frac{\partial w}{\partial y} \\
v(\rho E+p)-\frac{\eta}{R e}\left[u\left(\frac{\partial u}{\partial y}+\frac{\partial v}{\partial x}\right)+v\left(\frac{4}{3} \frac{\partial v}{\partial y}-\frac{2}{3} \frac{\partial u}{\partial x}\right)+w \frac{\partial w}{\partial y}\right]-\frac{\lambda}{\operatorname{PrRe}(\gamma-1) M_{\infty}^{2}} \frac{\partial T}{\partial y}
\end{array}\right) \\
& \mathbf{G}_{v z}=\left(\begin{array}{c}
0 \\
0 \\
-\frac{2}{3} \frac{\eta}{R e} \frac{\partial w}{\partial z} \\
\frac{\eta}{R e} \frac{\partial v}{\partial z} \\
\frac{\eta}{R e}\left[-\frac{2}{3} v \frac{\partial w}{\partial z}+w \frac{\partial v}{\partial z}\right]
\end{array}\right)
\end{aligned}
$$

$\mathbf{H}=\mathbf{H}^{\prime}-\mathbf{H}_{v z}$

$$
\mathbf{H}=\left(\begin{array}{c}
\rho w \\
\rho u w-\frac{1}{R e} \tau_{z x} \\
\rho v w-\frac{1}{R e} \tau_{z y} \\
\rho w^{2}+p-\frac{1}{R e} \tau_{z z} \\
w(\rho E+p)-\frac{1}{\operatorname{Re}}\left[u \tau_{z x}+v \tau_{z y}+w \tau_{z z}\right]-\frac{\lambda}{\operatorname{PrRe}(\gamma-1) M_{\infty}^{2}} \frac{\partial T}{\partial z}
\end{array}\right)
$$




$$
\mathbf{H}^{\prime}=\left(\begin{array}{c}
\rho w \\
\rho u w-\frac{\eta}{R e} \frac{\partial w}{\partial x} \\
\rho v w-\frac{\eta}{R e} \frac{\partial w}{\partial y} \\
\rho w^{2}+p-\frac{\eta}{R e}\left(-\frac{2}{3} \frac{\partial u}{\partial x}-\frac{2}{3} \frac{\partial v}{\partial y}\right) \\
\mathbf{H}_{v z}=\left(\begin{array}{c}
\eta \\
\left.w(\rho E+p)-\frac{\partial w}{\operatorname{Re}}+v \frac{\partial w}{\partial y}+w\left(-\frac{2}{3} \frac{\partial u}{\partial x}-\frac{2}{3} \frac{\partial v}{\partial y}\right)\right]
\end{array}\right) \\
0 \\
\frac{\eta}{R e} \frac{\partial u}{\partial z} \\
\frac{\eta}{R e} \frac{\partial v}{\partial z} \\
\frac{\eta}{R e} \frac{4}{3} \frac{\partial w}{\partial z} \\
\frac{\eta}{R e}\left[u \frac{\partial u}{\partial z}+v \frac{\partial v}{\partial z}+\frac{4}{3} w \frac{\partial w}{\partial z}\right]+\frac{\lambda}{\operatorname{PrRe}(\gamma-1) M_{\infty}^{2}} \frac{\partial T}{\partial z}
\end{array}\right)
$$

Appendix B. Computation of $\mathscr{J}_{v z}^{\prime}:$ modified fluxes $\widehat{\mathbf{F}}_{v z}, \widehat{\mathrm{G}}_{v z}$ and $\widehat{\mathbf{H}}_{v z}$

Full expressions of the modified fluxes $\widehat{\mathbf{F}}_{v z}, \widehat{\mathbf{G}}_{v z}$ and $\widehat{\mathbf{H}}_{v z}$ used in section 3.3.3 to compute the Jacobian matrix $\mathscr{J}_{v z}^{\prime}$ are given hereafter.

$$
\begin{aligned}
& \widehat{\mathbf{F}}_{v z}=\left(\begin{array}{c}
0 \\
-\frac{2}{3} \frac{\bar{\eta}}{R e} w \\
0 \\
\bar{\eta} \frac{R e}{R e} u \\
\frac{\bar{\eta}}{\operatorname{Re}}\left[-\bar{u} \frac{2}{3} w+\bar{w} u\right]
\end{array}\right) \\
& \widehat{\mathbf{G}}_{\nu z}=\left(\begin{array}{c}
0 \\
0 \\
-\frac{2}{3} \frac{\bar{\eta}}{R e} w \\
\frac{\bar{\eta}}{\operatorname{Re}} v \\
\frac{\bar{\eta}}{\operatorname{Re}}\left[-\frac{2}{3} \bar{v} w+\bar{w} v\right]
\end{array}\right) \\
& \widehat{\mathbf{H}}_{v z}=\left(\begin{array}{c}
0 \\
\frac{\bar{\eta}}{\operatorname{Re}} u \\
\frac{\bar{\eta}}{\operatorname{Re}} v \\
\frac{\bar{\eta}}{\operatorname{Re}} w \\
\frac{\bar{\eta}}{\operatorname{Re}}\left[\bar{u} u+\bar{v} v+\frac{4}{3} \bar{w} w\right]+\frac{\bar{\lambda}}{\operatorname{Pr} \operatorname{Re}(\gamma-1) M_{\infty}^{2}} T
\end{array}\right)
\end{aligned}
$$

\section{Appendix C. Explicit expression of norm matrices written in conservative variables}

Full expressions of norm matrices, associated with the discrete scalar products presented in section 3.5, are given for the kinetic energy of perturbations $\left(\mathbf{Q}_{\mathrm{KE}}\right)$ and the canonical norm of the forcing field $\left(\mathbf{Q}_{F}\right)$. The matrix $\mathbf{Q}_{\mathrm{KE}}$ is derived similarly to $\mathbf{Q}_{\mathrm{Chu}}$ in section 3.5, the latter actually containing the former. Its expression reads

$$
\mathbf{Q}_{\mathrm{KE}}=\frac{1}{2} \mathrm{~d} \Omega\left(\begin{array}{ccccc}
\frac{|\overline{\mathbf{u}}|^{2}}{\bar{\rho}} & -\frac{\bar{u}}{\bar{\rho}} & -\frac{\bar{v}}{\bar{\rho}} & 0 & 0 \\
-\frac{\bar{u}}{\bar{\rho}} & \frac{1}{\bar{\rho}} & 0 & 0 & 0 \\
-\frac{\bar{v}}{\bar{\rho}} & 0 & \frac{1}{\bar{\rho}} & 0 & 0 \\
0 & 0 & 0 & 0 & 0 \\
0 & 0 & 0 & 0 & 0
\end{array}\right)
$$


The matrix $\mathbf{Q}_{F}$ is associated to a canonical scalar product and can readily be expressed as

$$
\mathbf{Q}_{F}=\mathrm{d} \Omega\left(\begin{array}{lllll}
1 & 0 & 0 & 0 & 0 \\
0 & 1 & 0 & 0 & 0 \\
0 & 0 & 1 & 0 & 0 \\
0 & 0 & 0 & 1 & 0 \\
0 & 0 & 0 & 0 & 1
\end{array}\right)
$$

As noted in section 3.5, the numerical implementation of the above expression is achieved by building a block-diagonal matrix from these blocks, taking care of setting elementary volumes $\mathrm{d} \Omega_{i, j}$ and baseflow values for each point.

\section{Appendix D. Mesh convergence}

Mesh convergence of optimal gain computations presented in section 5.2 is examined. Mesh A $(1600 \times 81$, see Table D.6) is found sufficient for both streaks (low frequencies) and first mode (medium frequencies) computations (Fig. D.15). The height $L_{y}=9 \delta_{0}^{*}$ of the numerical domain is high enough to ensure the independence of the results with respect to this parameter. At higher frequencies, it is observed that mesh A is not fine enough as the streamwise wave number of the computed responses gets smaller and a strong velocity gradient is observed close to the plate (see Fig. 12). Thus, a finer mesh is used for $\omega>2.0$ to ensure the mesh convergence of the peak associated with the second mode. As shown in Fig. D.15, mesh $\mathrm{E}(2400 \times 108)$ is found sufficiently fine to compute this peak.
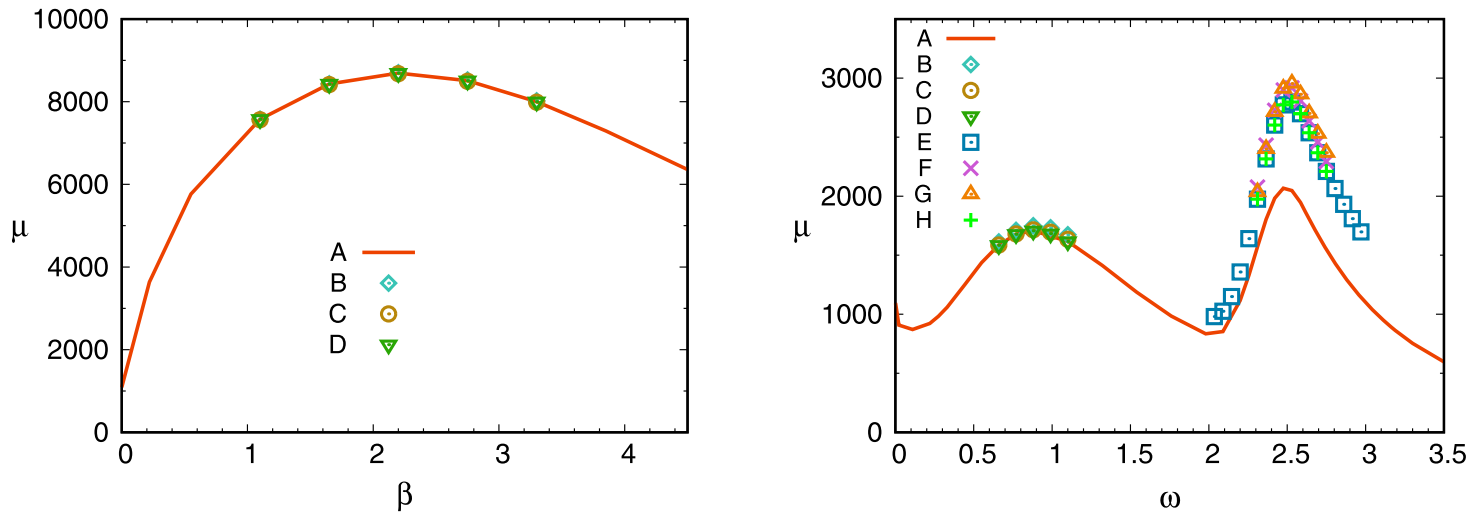

Fig. D.15. Mesh convergence (nomenclature of the different meshes is given in Table D.6). Left: optimal gain as a function of $\beta$ at $\omega=2 \times 10^{-3}$. Right: optimal gain as a function of $\omega$ at $\beta=0$.

Table D.6

Nomenclature of meshes used in Fig. D.15.

\begin{tabular}{llll}
\hline Mesh & $N_{x}$ & $N_{y}$ & $L_{y}$ \\
\hline A & 1600 & 81 & 9 \\
B & 1600 & 108 & 9 \\
C & 2400 & 81 & 9 \\
D & 1600 & 106 & 18 \\
E & 2400 & 108 & 9 \\
F & 2400 & 165 & 9 \\
G & 3200 & 108 & 9 \\
H & 2400 & 144 & 18 \\
\hline
\end{tabular}

\section{Appendix E. Computational costs}

Solving the linear systems involved in the computation of the Krylov vector (Algorithm 1 in section 3.5) is the bottleneck in terms of both CPU time and RAM requirements. Besides, it cannot be known a priori how many Krylov vectors are needed to solve the eigenvalue problem in equation (48) until a residual equal to $10^{-3}$ is reached. In practice, it is observed that a minimum of 3 vectors and a maximum of 12 vectors are needed and that large values of the optimal gain require fewer number of Krylov vectors. Numerical costs involved in the optimal gain computations of the boundary layer at $M=4.5$ (section 5.2) are reported in Table E.7. The 3D perturbations solver requires approximately twice the time and less than 
Table E.7

CPU time and maximum RAM required to compute one Krylov vector to solve the eigenvalue problem (48) using mesh A (see Table D.6). These computations were conducted using Intel Xeon(R) CPU E5-2630 v2@ 2.60GHz.

\begin{tabular}{lll}
\hline Solver & CPU time & maximum RAM \\
\hline 2D perturbations & $42 \mathrm{~min}$ & $7.31 \mathrm{~GB}$ \\
3D perturbations & $88 \mathrm{~min}$ & $12.5 \mathrm{~GB}$ \\
\hline
\end{tabular}

twice the maximum RAM that is needed to compute the optimal gain for 2D perturbations. Hence, the numerical costs lie in the same order of magnitude for both solvers, which means that if one can afford a 2D perturbations computation, one can generally afford a 3D perturbations computation.

\section{References}

[1] C. Mettot, F. Renac, D. Sipp, Computation of eigenvalue sensitivity to base flow modifications in a discrete framework: application to open-loop control, J. Comput. Phys. 269 (2014) 234-258.

[2] P. Huerre, P.A. Monkewitz, Local and global instabilities in spatially developing flows, Annu. Rev. Fluid Mech. 22 (1) (1990) $473-537$.

[3] P.J. Schmid, D.S. Henningson, Stability and Transition in Shear Flows, vol. 142, Springer Science \& Business Media, 2012.

[4] L.M. Mack, A numerical study of the temporal eigenvalue spectrum of the Blasius boundary layer, J. Fluid Mech. 73 (3) (1976) 497-520.

[5] P.A. Monkewitz, The absolute and convective nature of instability in two-dimensional wakes at low Reynolds numbers, Phys. Fluids 31 (5) (1988) 999-1006.

[6] A. Michalke, On spatially growing disturbances in an inviscid shear layer, J. Fluid Mech. 23 (3) (1965) 521-544.

[7] P. Huerre, P.A. Monkewitz, Absolute and convective instabilities in free shear layers, J. Fluid Mech. 159 (1985) 151-168.

[8] L. Lees, C. Lin, Investigation of the Stability of the Laminar Boundary Layer in a Compressible Fluid, Tech. Rep., National Advisory Committee for Aeronautics, Washington, DC, 1946.

[9] D.W. Dunn, C.C. Lin, On the Stability of the Laminar Boundary Layer in a Compressible Fluid, Ph.D. thesis, Massachusetts Institute of Technology, 1953.

[10] L.M. Mack, Numerical Calculation of the Stability of the Compressible, Laminar Boundary Layer, Jet Propulsion Laboratory, California Institute of Technology, 1960.

[11] L.M. Mack, Boundary-Layer Linear Stability Theory, Tech. Rep., DTIC Document, 1984.

[12] H.B. Squire, On the stability for three-dimensional disturbances of viscous fluid flow between parallel walls, Proc. R. Soc. Lond., a Contain. Pap. Math. Phys. Character 142 (847) (1933) 621-628.

[13] L.M. Mack, Linear stability theory and the problem of supersonic boundary-layer transition, AIAA J. 13 (3) (1975) 278-289.

[14] J. Kendall, Wind tunnel experiments relating to supersonic and hypersonic boundary-layer transition, AIAA J. 13 (3) (1975) 290-299.

[15] K. Stetson, R. Kimmel, On hypersonic boundary-layer stability, in: 30th Aerospace Sciences Meeting and Exhibit, $1992,737$.

[16] N.M. El-Hady, Nonparallel instability of supersonic and hypersonic boundary layers, Phys. Fluids A, Fluid Dyn. 3 (9) (1991) $2164-2178$.

[17] C.L. Chang, M. Malik, Non-parallel stability of compressible boundary layers, in: 23rd Fluid Dynamics, Plasmadynamics, and Lasers Conference, 1993, p. 2912.

[18] B.F. Farrell, Optimal excitation of perturbations in viscous shear flow, Phys. Fluids 31 (8) (1988) 2093.

[19] A. Hanifi, P.J. Schmid, D.S. Henningson, Transient growth in compressible boundary layer flow, Phys. Fluids (1994-present) 8 (3) (1996) $826-837$.

[20] A. Tumin, E. Reshotko, Spatial theory of optimal disturbances in boundary layers, Phys. Fluids 13 (7) (2001) 2097-2104.

[21] A. Tumin, E. Reshotko, Optimal disturbances in compressible boundary layers, AIAA J. 41 (12) (2003) 2357-2363.

[22] S. Zuccher, A. Tumin, E. Reshotko, Parabolic approach to optimal perturbations in compressible boundary layers, J. Fluid Mech. 556 (2006) 189-216.

[23] D. Tempelmann, A. Hanifi, D.S. Henningson, Spatial optimal growth in three-dimensional compressible boundary layers, J. Fluid Mech. 704 (2012) 251-279.

[24] T. Herbert, Parabolized stability equations, Annu. Rev. Fluid Mech. 29 (1) (1997) 245-283.

[25] P. Paredes, M.M. Choudhari, F. Li, C.L. Chang, Transient growth analysis of compressible boundary layers with parabolized stability equations, AIAA Pap. 51 (2016) 2016.

[26] P. Paredes, M.M. Choudhari, F. Li, C.L. Chang, Optimal growth in hypersonic boundary layers, AIAA J. 54 (10) (2016) 3050-3061.

[27] V. Theofilis, Advances in global linear instability analysis of nonparallel and three-dimensional flows, Prog. Aerosp. Sci. 39 (4) (2003) $249-315$.

[28] G.A. Bres, T. Colonius, Three-dimensional instabilities in compressible flow over open cavities, J. Fluid Mech. 599 (2008) 309-339.

[29] J.C. Robinet, Bifurcations in shock-wave/laminar-boundary-layer interaction: global instability approach, J. Fluid Mech. 579 (2007) 85-112.

[30] J. Crouch, A. Garbaruk, D. Magidov, Predicting the onset of flow unsteadiness based on global instability, J. Comput. Phys. 224 (2) (2007) 924-940.

[31] D. Sipp, O. Marquet, P. Meliga, A. Barbagallo, Dynamics and control of global instabilities in open-flows: a linearized approach, Appl. Mech. Rev. 63 (3) (2010) 030801.

[32] U. Ehrenstein, F. Gallaire, On two-dimensional temporal modes in spatially evolving open flows: the flat-plate boundary layer, J. Fluid Mech. 536 (2005) 209-218.

[33] F. Alizard, J.C. Robinet, Spatially convective global modes in a boundary layer, Phys. Fluids 19 (11) (2007) 114105.

[34] A. Monokrousos, E. Åkervik, L. Brandt, D.S. Henningson, Global three-dimensional optimal disturbances in the blasius boundary-layer flow using timesteppers, J. Fluid Mech. 650 (2010) 181-214.

[35] D. Sipp, O. Marquet, Characterization of noise amplifiers with global singular modes: the case of the leading-edge flat-plate boundary layer, Theor. Comput. Fluid Dyn. 27 (5) (2013) 617-635.

[36] P.J. Schmid, Nonmodal stability theory, Annu. Rev. Fluid Mech. 39 (2007) 129-162.

[37] J.M. Chomaz, Global instabilities in spatially developing flows: non-normality and nonlinearity, Annu. Rev. Fluid Mech. 37 (2005) $357-392$.

[38] O. Marquet, M. Lombardi, J.M. Chomaz, D. Sipp, L. Jacquin, Direct and adjoint global modes of a recirculation bubble: lift-up and convective nonnormalities, J. Fluid Mech. 622 (2009) 1-21.

[39] M. Landahl, A note on an algebraic instability of inviscid parallel shear flows, J. Fluid Mech. 98 (2) (1980) 243-251.

[40] W.M. Orr, The stability or instability of the steady motions of a perfect liquid and of a viscous liquid. Part I: a perfect liquid, in: Proceedings of the Royal Irish Academy. Section A: Mathematical and Physical Sciences, in: JSTOR, vol. 27, 1907, pp. 9-68.

[41] J.W. Nichols, S.K. Lele, Global modes and transient response of a cold supersonic jet, J. Fluid Mech. 669 (2011) 225-241.

[42] X. Garnaud, L. Lesshafft, P. Schmid, P. Huerre, Modal and transient dynamics of jet flows, Phys. Fluids 25 (4) (2013) 044103. 
[43] F. Sartor, C. Mettot, R. Bur, D. Sipp, Unsteadiness in transonic shock-wave/boundary-layer interactions: experimental investigation and global stability analysis, J. Fluid Mech. 781 (2015) 550-577.

[44] W. Sutherland, LII. The viscosity of gases and molecular force, Lond. Edinb. Dublin Philos. Mag., J. Sci. 36 (223) (1893) $507-531$.

[45] E.F. Toro, Riemann Solvers and Numerical Methods for Fluid Dynamics: A Practical Introduction, Springer Science \& Business Media, 2013.

[46] M.S. Liou, A sequel to AUSM: AUSM+, J. Comput. Phys. 129 (2) (1996) 364-382.

[47] J.P. Boin, J.C. Robinet, C. Corre, H. Deniau, 3d steady and unsteady bifurcations in a shock-wave/laminar boundary layer interaction: a numerical study, Theor. Comput. Fluid Dyn. 20 (3) (2006) 163-180.

[48] A. Jameson, Time dependent calculations using multigrid, with applications to unsteady flows past airfoils and wings, AIAA Pap. 1596 (1991) 1991.

[49] C. Mettot, Linear Stability, Sensitivity, and Passive Control of Turbulent Flows Using Finite Differences, Ph.D. thesis, Palaiseau, Ecole polytechnique, 2013.

[50] D.A. Knoll, D.E. Keyes, Jacobian-free Newton-Krylov methods: a survey of approaches and applications, J. Comput. Phys. 193 (2) (2004) $357-397$.

[51] B.T. Chu, On the energy transfer to small disturbances in fluid flow (part i), Acta Mech. 1 (3) (1965) $215-234$.

[52] V. Theofilis, Global linear instability, Annu. Rev. Fluid Mech. 43 (2011) 319-352.

[53] S. Balay, S. Abhyankar, M.F. Adams, J. Brown, P. Brune, K. Buschelman, et al., PETSc Web page, http://www.mcs.anl.gov/petsc, 2016.

[54] P.R. Amestoy, I.S. Duff, J.Y. L'Excellent, J. Koster, A fully asynchronous multifrontal solver using distributed dynamic scheduling, SIAM J. Matrix Anal. Appl. 23 (1) (2001) 15-41.

[55] S. Balay, S. Abhyankar, M.F. Adams, J. Brown, P. Brune, K. Buschelman, et al., PETSc users manual, Tech. Rep. ANL-95/11 - Revision 3.7, Argonne National Laboratory, 2016.

[56] V. Hernández, J.E. Román, A. Tomás, V. Vidal, Krylov-Schur Methods in SLEPc, Tech. Rep. STR-7, Universitat Politecnica de Valencia, 2007.

[57] N. Hildebrand, A. Dwivedi, J.W. Nichols, M.R. Jovanović, G.V. Candler, Simulation and stability analysis of oblique shock-wave/boundary-layer interactions at mach 5.92, Phys. Rev. Fluids 3 (1) (2018) 013906.

[58] S. Özgen, S.A. Kırcalı, Linear stability analysis in compressible, flat-plate boundary-layers, Theor. Comput. Fluid Dyn. 22 (1) (2008) 1-20.

[59] L.M. Mack, Review of linear compressible stability theory, in: Stability of Time Dependent and Spatially Varying Flows, Springer, 1987 , pp. 164-187.

[60] T. Ellingsen, E. Palm, Stability of linear flow, Phys. Fluids 18 (4) (1975) 487-488.

[61] G. Erlebacher, M. Hussaini, Numerical experiments in supersonic boundary-layer stability, Phys. Fluids A, Fluid Dyn. 2 (1) (1990) 94-104.

[62] M.R. Malik, P. Balakumar, Acoustic receptivity of mach 4.5 boundary layer with leading-edge bluntness, Theor. Comput. Fluid Dyn. 21 (5) (2007) $323-342$.

[63] A. Towne, G.A. Bres, S.K. Lele, A statistical jet-noise model based on the resolvent framework, in: 23rd AIAA/CEAS Aeroacoustics Conference, 2017, 3706. 